\title{
A RECENT GEOMORPHIC AND PEDOLOGICAL STUDIES ON MENOUF PROVINCE SOILS, MENOUFIA GOVERNORATE, EGYPT
}

\author{
M.S. Amira (1), F.E. Abu Agwa(1), E.A. Abu Hussien(1), A.B. Belal(2) \\ and Asmaa M. Soliman (') \\ (1) Soil Sci. Dept., Fac. Agric. Menoufia univ . \\ (2) National Authority for Remote Sensing and Space Sciences (NARSS)
}

Received: Oct. 26,2020

Accepted: Nov. 4,2020

\begin{abstract}
The current work was performed in 2018 aiming to study the geomorphological and pedological characteristics as well as classification and capability evaluation for soils of Menouf province area, Menoufia governorate, Egypt. The integration of Remote Sensing (RS) and Geographic Information System (GIS) techniques was used to achieve this work.
\end{abstract}

The geomorphic map produced by processing and identifying the Landsat image using RS and GIS technology indicated that, the main landscape unit in the studied area is Alluvial Plain includes nine landforms namely: high terraces (19.4\% of the studied area), medium terraces (15.3\%), low terraces (24.6\%), over flow basin (25.6\%), decantation basin (7.9\%) in addition to meandering belt, depression, levee, and island (with small areas).

Twenty-four soil profiles were chosen to represent the different landforms. The land and site features are observed and registered. The soil profiles were dug, morphologically described, and then samples were collected representing the subsequent layers in each profile for integrated physical and chemical analyses.

The studied area has almost flat topography with deep soil profiles and freely well drained. These soils have loam to sandy clay loam texture with moderate medium sub angular to angular blocky structure.

The analytical data revealed that, the studied soils are moderately alkaline, non-saline and haven't sodicity effect. The soils are slightly calcareous having very slight gypsum content. Organic matter (OM) is low and decreases with depth. The cation exchange capacity (CEC) is correlated to the fine fractions and OM contents in these soils.

The studied soil profiles haven't any diagnostic horizons, therefore they were classified up to sub great group level under Entisols order mainly as Typic Torriorthents.

The land capability evaluation indicated that, about $48 \%$ of the studied soils have a Good capability class (C2) and the rest (52\%) are considered as a Fair (C3) one.

Key words: RS, GIS, geomorphic units, pedological features, soil classification, land capability evaluation.

\section{INTRODUCTION}

Menoufia governorate is one from the main agricultural governorates of Egypt. It is located in the southern part of the Nile Delta. It has 10 provinces with a total area of about $2,543.03 \mathrm{~km}^{2}$, and population of about 4,366,000 according to the governorate estimation in January 2018 (CAPMS, 2018). Menouf province (the studied area) is one of the oldest provinces and Menouf city is the old capital of the governorate. Agriculture is generally the main activity of the population due to its fertile land in the Nile Delta. The governorate is famous for the production of crops like cotton, maize and wheat as well as vegetable crops such as potatoes and green beans of which a large part from these crops is exported. Agricultural land is irrigated with Nile 
M.S. Amira, et al.,

water from the Rosetta and Damietta branches.

Remote sensing (RS) is now recognized as an important tool in monitoring and managing natural resources (Lillesand and Kiefer, 2007). They added that RS technique is one of the important methods that used for soil survey, mapping and environmental investigation.

ESRI (2003) stated that, geographic information system (GIS) is a system for the management, analysis, and displaying geographic information, which is represented by a series of geographic datasets that model geography using simple, generic data structures.

Integration of RS and GIS play a major role in both soil survey and soil mapping applications. The development of methods to map soil properties using optical RS data in combination with field measurements has been the objective of several studies during the last decade (Dehaan and Taylor, 2003).

This investigation was performed in 2018 to furnish a recent study on geomorphological and pedological features including classification and capability evaluation of the soils in Menouf province using the integration of remote sensing (RS) and geographic information system (GIS) techniques. This work could present important information served for promising plans of improvement and management of these soils.

\section{MATERIALS AND METHODS}

\section{Study area}

The study area (Menouf province) is located at the middle west of the governorate and east of Rosetta branch. It lies between longitudes $30^{\circ} 50^{\circ}$ and $31^{\circ}$ $00^{\circ} \mathrm{E}$ and latitudes $30^{\circ} 20^{\circ}$ and $30^{\circ} 35^{\circ} \mathrm{N}$, with an area of $225.336 \mathrm{~km} 2$ (53582.3 feddans), Fig (1). The studied area are characterized by a hot dry summer and warm winter with few rainfalls.

\section{Producing geomorphic map for the study area}

The digital elevation model (DEM) of the study area was extracted from the Shuttle Radar Topography Mission (SRTM) and a topographic map with a scale of 1:25,000 covering the study area using Arc-GIS 10.4 software (ESRI, 2003). The Landsat 8 (path 177 I row 39) image acquired in 2018 and SRTM data were processed in ENVI 5.1 software (ITT, 2012) to identify the landforms of the studied area according to the approach developed by Dobos et al. (2002). The map legend was designed according to Zinck and Valenzuela (1990). ArcMap 10.4 software was used to display and produce geomorphic map with help of field observations (ESRI, 2014).

\section{Field Work.}

Reconnaissance survey was conducted throughout the investigated area in order to acquire an appreciation of its broad soil patterns and characteristic landscape. The primary mapping units resulting from analysis of the DEM and interpretation information gained during unsupervised classification Landsat images were verified.

Longitudes and latitudes as well as elevation are defined in the field by using GPS "System Corporation MAGELLAN"GPS NAV DLX-10 TM for recognizing and soil profiles locations within the studied area.

Twenty-four soil profiles were chosen to represent the landform units in the studied area (Fig, 2). Detailed morphological description of these soil profiles was recorded on the basis outlined by FAO (2006). Soil samples were collected based on the vertical variations of each soil profile for the laboratory analyses of soil physical and chemical properties. 


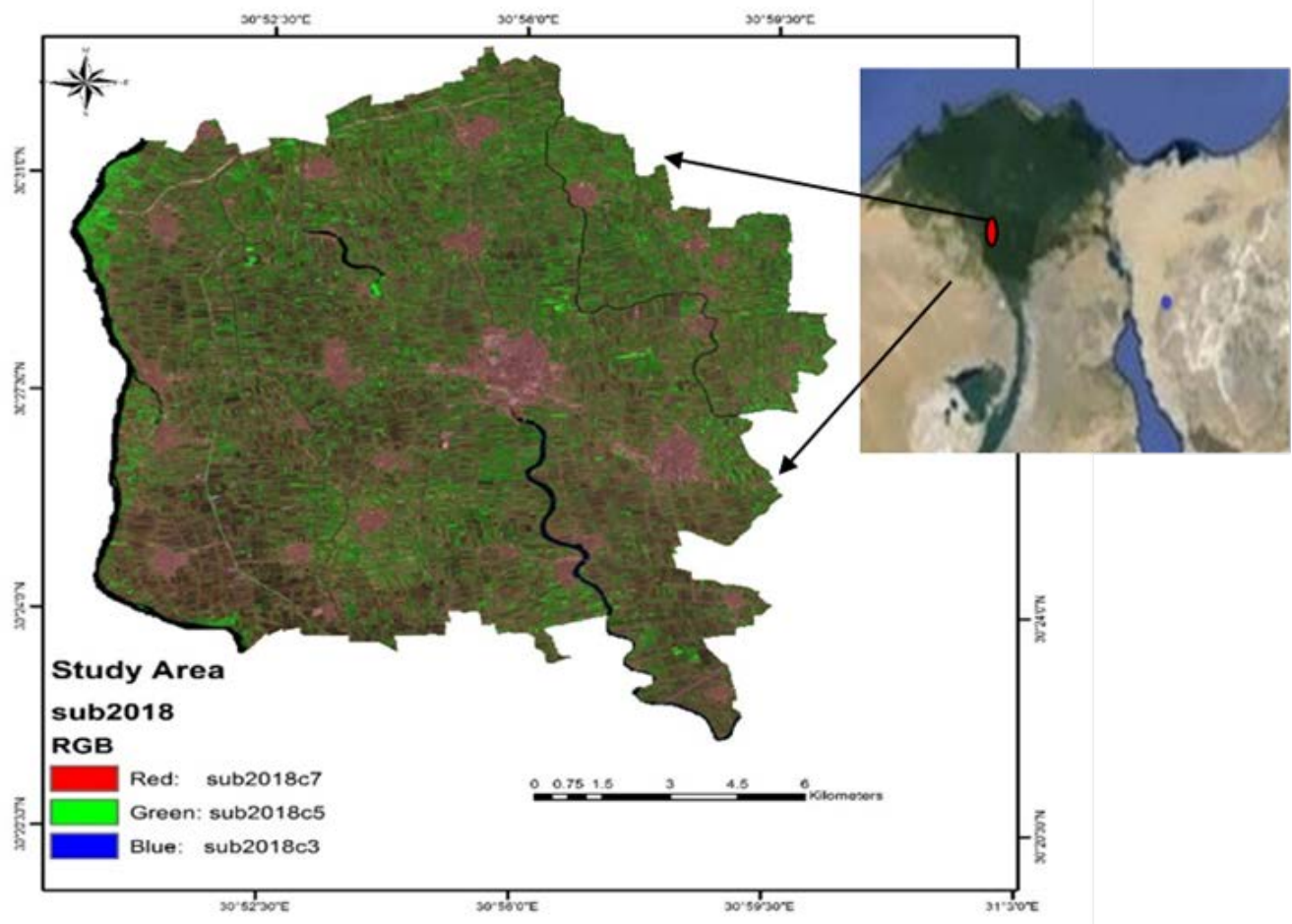

Fig (1): Location map of Menouf province, study area.

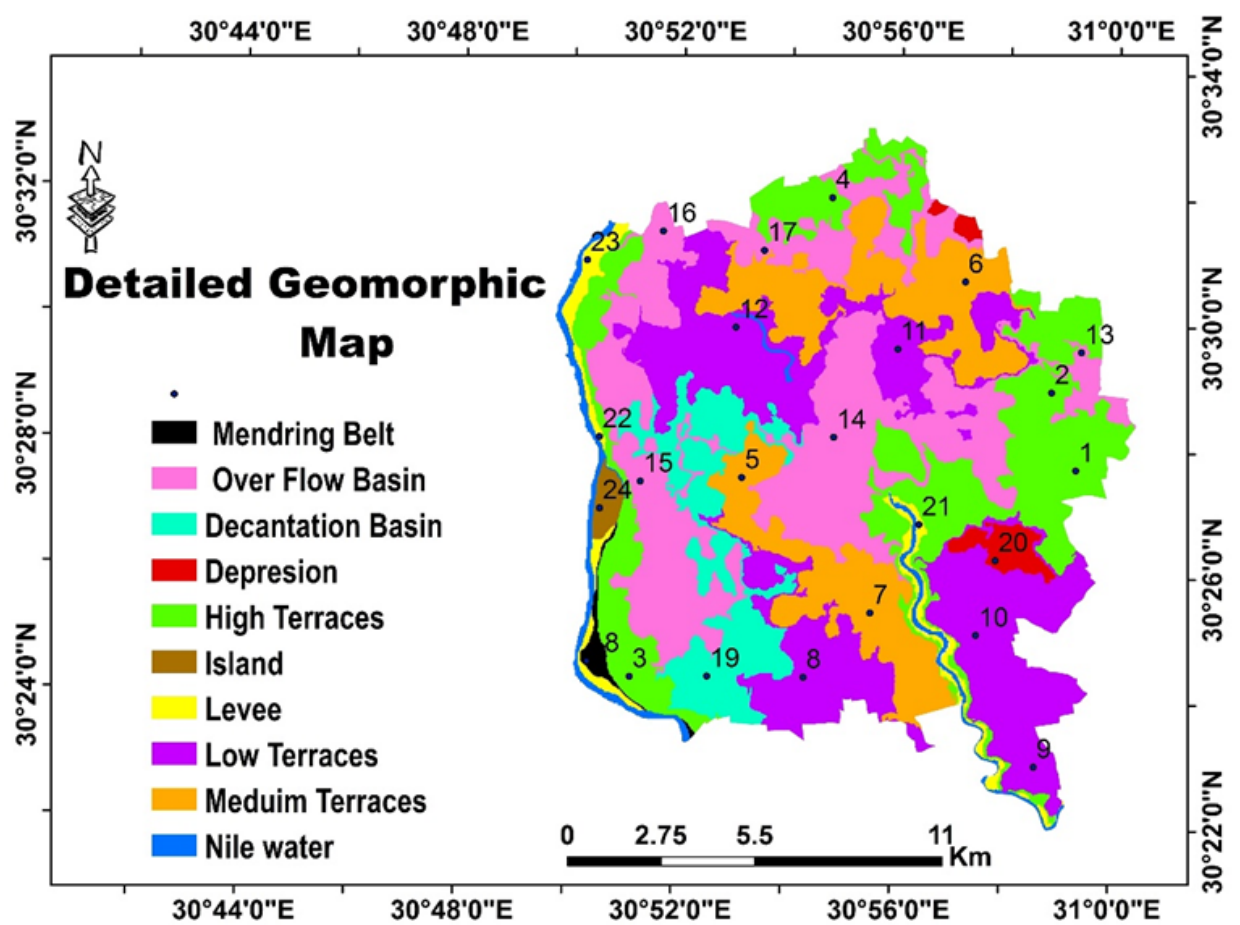

Fig (2): Detailed geomorphic map and locations of soil profiles in the study area.

\section{Laboratory Analyses}

Particle size distribution, electrical conductivity (EC), pH, organic matter
(OM), calcium carbonate $\left(\mathrm{CaCO}_{3}\right)$, gypsum contents, cation exchange capacity (CEC) and exchangeable $\mathrm{Na}^{+}$percentage (ESP), 
M.S. Amira, et al.,

were determined according to Burt and Soil Survey Staff (2014). The weighted profile mean (w.p.m.) of each soil property was calculated for the studied profiles.

\section{Soil classification}

The soils of the studied area were classified up to sub great group level based on Soil Survey Staff (2014).

\section{Land Evaluation}

Land capability classification was carried out using the Applied System of Land Evaluation (ASLE software) developed by Ismail et al. (2005). ASLE software is inserting of soil database and calculates possible indices combinations between the major land properties. These properties are irrigation system, number of layers and layers depths, physical properties (e.g. clay content, available water, profile depth, landform, slope and level of surface) and chemical properties (e.g. pH, soil salinity, gypsum and carbonate contents),

\section{RESULTUS AND DISCUSSION}

\section{Geomorphology}

Based on the integration of RS and GIS works as well as the satellite image interpretation, the investigated area could be considered mainly as alluvial plain geomorphic unit with nine detailed landforms (Fig, 2 and Table, 1). These landforms are high terraces (profiles 1,2,3 and 4), medium terraces (profiles 5,6 and 7 ), low terraces (profiles $8,9,10,11$ and 12 ), over flow basin (profiles 13,14,15,16 and 17), meandering belt (profile 18), decantation basin (profile 19), depression (profile 20), levee (profiles 21, 22 and 23), and island (profile 24).

\section{Soil Morphology}

The morphological features of the studied soils presented in Table (2) revealed that, the elevation of the studied soils is between $7 \mathrm{~m}$ above sea level (at the Levee) to $12 \mathrm{~m}$ a.s.l. (at the High Terraces). The soils have almost flat topography. All studied soils are deep and well drained. The main hue notation of studied soil color is around brown degrees (10YR). These soils have mainly Loam or sandy clay loam to clay loam texture throughout their depths with mostly moderate medium angular to subangular blocky structure. They are slightly to moderately calcareous having mostly hard to extremely hard (dry) and firm to extremely firm (moist) consistency. The most studied soils are cultivated with field or horticultural crops.

Table (1): Areas of the landforms of the study area.

\begin{tabular}{|l|l|c|c|}
\hline \multirow{2}{*}{ Geomorphic unit } & \multicolumn{1}{c|}{ Landforms } & \multicolumn{2}{c|}{ Area } \\
\cline { 2 - 4 } & \multicolumn{1}{c|}{$\mathrm{km}^{2}$} & $\%$ \\
\hline \multirow{4}{*}{ Alluvial plain } & High Terraces & 43.731 & 19.406 \\
\cline { 2 - 4 } & Medium Terraces & 34.606 & 15.357 \\
\cline { 2 - 4 } & Low Terraces & 55.470 & 24.617 \\
\cline { 2 - 4 } & Over Flow Basin & 57.641 & 25.579 \\
\cline { 2 - 4 } & Meandering belt & 1.223 & 0.543 \\
\cline { 2 - 4 } & Decantation Basin & 17.808 & 7.903 \\
\cline { 2 - 4 } & Depression & 3.280 & 1.456 \\
\cline { 2 - 4 } & Levee & 5.763 & 2.558 \\
\cline { 2 - 4 } & Island & 1.413 & 0.627 \\
\hline Reference term & Water bodies & 4.401 & 1.953 \\
\hline Total & & 225.330 & 100,000 \\
\hline
\end{tabular}


A recent geomorphic and pedological studies on Menouf province soils,

Table (2): Morphological features of the studied soil profiles.

\begin{tabular}{|c|c|c|c|c|c|c|c|c|c|}
\hline \multirow{2}{*}{\begin{tabular}{|c} 
Geomor \\
phic \\
units \\
\end{tabular}} & \multirow{2}{*}{$\begin{array}{c}\text { Profile } \\
\text { No. }\end{array}$} & \multirow{2}{*}{$\begin{array}{l}\text { Elevation } \\
\text { m asl }\end{array}$} & \multirow{2}{*}{$\begin{array}{l}\text { Depth } \\
\text { cm }\end{array}$} & \multicolumn{2}{|c|}{ Color } & \multirow[b]{2}{*}{ Structure $^{1}$} & \multicolumn{2}{|c|}{ Consistence $^{2}$} & \multirow[b]{2}{*}{ Boundary } \\
\hline & & & & Dry & Moist & & Dry & Moist & \\
\hline \multirow{4}{*}{$\begin{array}{l}\text { d } \\
\frac{\pi}{\pi} \\
\frac{\pi}{0} \\
1 \\
\frac{5}{5} \\
\frac{5}{1}\end{array}$} & 1 & +11 & \begin{tabular}{|c|}
$0-30$ \\
$30-60$ \\
$60-90$ \\
$90-110$ \\
$110-138$ \\
\end{tabular} & $\begin{array}{l}\text { 10YR 4/3 } \\
\text { 10YR 4/3 } \\
\text { 10YR 5/3 } \\
\text { 10YR 4/3 } \\
\text { 10YR 4/3 }\end{array}$ & $\begin{array}{l}3 / 2 \\
3 / 3 \\
3 / 3 \\
3 / 3 \\
3 / 3 \\
\end{array}$ & $\begin{array}{l}2 \mathrm{~m} \mathrm{sbk} \\
2 \mathrm{~m} \mathrm{bk} \\
2 \mathrm{~m} \mathrm{sbk} \\
2 \mathrm{~m} \mathrm{sbk} \\
2 \mathrm{~m} \mathrm{sbk}\end{array}$ & $\begin{array}{c}\text { S hard } \\
\text { ex hard } \\
\text { hard } \\
\text { hard } \\
\text { hard }\end{array}$ & $\begin{array}{c}\text { firm } \\
v \text { firm } \\
v \text { firm } \\
v \text { firm } \\
\text { firm } \\
\end{array}$ & $\begin{array}{c}\text { diffuse } \\
\text { gradual s } \\
\text { diffuse } \\
\text { diffuse } \\
- \\
\end{array}$ \\
\hline & 2 & +12 & \begin{tabular}{|c|}
$0-30$ \\
$30-60$ \\
$60-90$ \\
$90-115$ \\
$115-150$ \\
\end{tabular} & $\begin{array}{l}\text { 10YR 5/3 } \\
\text { 10YR 5/3 } \\
\text { 10YR 5/3 } \\
\text { 10YR 6/3 } \\
\text { 10YR 6/3 } \\
\end{array}$ & $\begin{array}{l}4 / 3 \\
4 / 3 \\
4 / 3 \\
4 / 3 \\
4 / 3 \\
\end{array}$ & $\begin{array}{l}2 \mathrm{~m} \mathrm{bk} \\
2 \mathrm{~m} \mathrm{sbk} \\
2 \mathrm{~m} \mathrm{sbk} \\
2 \mathrm{~m} \mathrm{sbk} \\
2 \mathrm{~m} \mathrm{sbk}\end{array}$ & $\begin{array}{l}\text { ex hard } \\
\text { ex hard } \\
\text { ex hard } \\
v \text { hard } \\
s \text { hard }\end{array}$ & $\begin{array}{c}\text { firm } \\
\text { friable } \\
\text { firm } \\
\text { friable } \\
\text { v friable }\end{array}$ & $\begin{array}{c}\text { diffuse } \\
\text { diffuse } \\
\text { gradual s } \\
\text { diffuse } \\
- \\
\end{array}$ \\
\hline & 3 & +11 & \begin{tabular}{|c|}
$0-30$ \\
$30-60$ \\
$60-90$ \\
$90-120$ \\
$120-150$ \\
\end{tabular} & $\begin{array}{l}\text { 10YR 4/3 } \\
\text { 10YR 4/2 } \\
\text { 10YR 4/2 } \\
\text { 10YR 4/2 } \\
\text { 10YR 6/3 }\end{array}$ & $\begin{array}{l}4 / 3 \\
3 / 2 \\
3 / 2 \\
3 / 2 \\
4 / 3 \\
\end{array}$ & $\begin{array}{c}2 \mathrm{~m} \mathrm{sbk} \\
2 \mathrm{~m} \mathrm{bk} \\
2 \mathrm{~m} \mathrm{sbk} \\
2 \mathrm{~m} \mathrm{sbk} \\
2 \mathrm{~m} \mathrm{sbk}\end{array}$ & $\begin{array}{l}\text { v hard } \\
\text { ex hard } \\
\text { ex hard } \\
\text { ex hard } \\
\text { soft }\end{array}$ & $\begin{array}{c}\text { firm } \\
\text { ex firm } \\
\text { ex firm } \\
\text { v firm } \\
\text { soft }\end{array}$ & $\begin{array}{c}\text { gradual s } \\
\text { diffuse } \\
\text { diffuse } \\
\text { gradual s } \\
-\end{array}$ \\
\hline & 4 & +12 & $\begin{array}{c}0-30 \\
30-60 \\
60-90 \\
90-120 \\
120-135 \\
\end{array}$ & $\begin{array}{l}\text { 10YR 4/2 } \\
\text { 10YR 4/2 } \\
\text { 10YR 4/2 } \\
\text { 10YR 4/2 } \\
\text { 10YR 4/2 }\end{array}$ & $\begin{array}{l}3 / 2 \\
3 / 2 \\
3 / 2 \\
3 / 2 \\
3 / 2 \\
\end{array}$ & $\begin{array}{c}2 \mathrm{~m} \mathrm{sbk} \\
2 \mathrm{~m} \mathrm{bk} \\
2 \mathrm{~m} \mathrm{sbk} \\
2 \mathrm{~m} \mathrm{sbk} \\
2 \mathrm{~m} \mathrm{sbk}\end{array}$ & $\begin{array}{l}\text { ex hard } \\
\text { ex hard } \\
\text { ex hard } \\
\text { ex hard } \\
\text { ex hard }\end{array}$ & $\begin{array}{c}\text { ex firm } \\
\text { ex firm ex } \\
\text { firm } \\
\text { ex firm } \\
\text { ex firm } \\
\end{array}$ & $\begin{array}{c}\text { diffuse } \\
\text { diffuse } \\
\text { diffuse } \\
\text { diffuse } \\
-\end{array}$ \\
\hline \multirow{3}{*}{ 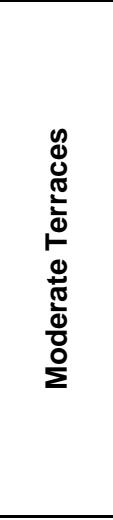 } & 5 & +10 & $\begin{array}{c}0-30 \\
30-60 \\
60-90 \\
90-120 \\
120-150 \\
\end{array}$ & $\begin{array}{l}\text { 10YR 4/3 } \\
\text { 10YR 4/3 } \\
\text { 10YR 5/2 } \\
\text { 10YR 5/2 } \\
\text { 10YR 5/2 } \\
\end{array}$ & $\begin{array}{l}3 / 2 \\
3 / 2 \\
3 / 2 \\
4 / 2 \\
3 / 2 \\
\end{array}$ & $\begin{array}{l}2 \mathrm{~m} \mathrm{bk} \\
2 \mathrm{~m} \mathrm{bk} \\
2 \mathrm{~m} \mathrm{bk} \\
2 \mathrm{~m} \mathrm{bk} \\
2 \mathrm{~m} \mathrm{bk} \\
\end{array}$ & $\begin{array}{l}\text { ex hard } \\
\text { ex hard } \\
\text { ex hard } \\
\text { ex hard } \\
\text { ex hard }\end{array}$ & $\begin{array}{c}\text { firm } \\
\text { firm } \\
\text { firm } \\
\text { ex firm } \\
\text { ex firm } \\
\end{array}$ & $\begin{array}{c}\text { gradual s } \\
\text { gradual s } \\
\text { diffuse } \\
\text { diffuse } \\
-\end{array}$ \\
\hline & 6 & +10 & $\begin{array}{c}0-30 \\
30-60 \\
60-90 \\
90-120 \\
120-150\end{array}$ & $\begin{array}{l}\text { 10YR 5/2 } \\
\text { 10YR 5/2 } \\
\text { 10YR 4/2 } \\
\text { 10YR 5/2 } \\
\text { 10YR 4/2 }\end{array}$ & $\begin{array}{l}4 / 2 \\
4 / 2 \\
3 / 2 \\
4 / 2 \\
3 / 2\end{array}$ & $\begin{array}{l}2 \mathrm{~m} \mathrm{bk} \\
2 \mathrm{~m} \mathrm{bk} \\
2 \mathrm{~m} \mathrm{bk} \\
2 \mathrm{~m} \mathrm{bk} \\
2 \mathrm{~m} \mathrm{bk}\end{array}$ & $\begin{array}{l}\text { ex hard } \\
\text { ex hard } \\
\text { ex hard } \\
\text { ex hard } \\
\text { ex hard }\end{array}$ & $\begin{array}{l}\text { ex firm } \\
\text { ex firm } \\
\text { ex firm } \\
\text { firm } \\
\text { v firm }\end{array}$ & $\begin{array}{c}\text { diffuse } \\
\text { gradual s } \\
\text { gradual s } \\
\text { gradual s } \\
-\end{array}$ \\
\hline & 7 & +10 & $\begin{array}{c}0-30 \\
30-60 \\
60-90 \\
90-120 \\
120-150 \\
\end{array}$ & $\begin{array}{l}\text { 10YR 5/3 } \\
\text { 10Y R 5/2 } \\
\text { 10YR 5/2 } \\
\text { 10YR 5/2 } \\
\text { 10YR 5/2 } \\
\end{array}$ & $\begin{array}{l}3 / 3 \\
4 / 2 \\
4 / 2 \\
4 / 2 \\
4 / 2 \\
\end{array}$ & $\begin{array}{l}2 \mathrm{mbk} \\
2 \mathrm{~m} \mathrm{bk} \\
2 \mathrm{~m} \mathrm{bk} \\
2 \mathrm{~m} \mathrm{bk} \\
2 \mathrm{~m} \mathrm{bk} \\
\end{array}$ & $\begin{array}{l}\text { ex hard } \\
\text { ex hard } \\
\text { ex hard } \\
\text { ex hard } \\
\text { ex hard }\end{array}$ & $\begin{array}{c}\text { firm } \\
\text { v firm } \\
\text { v firm } \\
\text { v firm } \\
\text { ex firm } \\
\end{array}$ & $\begin{array}{c}\text { gradual s } \\
\text { diffuse } \\
\text { diffuse } \\
\text { diffuse } \\
- \\
\end{array}$ \\
\hline \multirow{5}{*}{ 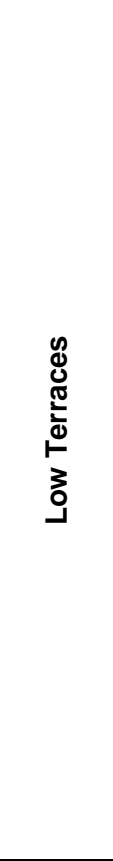 } & 8 & +8 & $\begin{array}{c}0-30 \\
30-70 \\
70-95 \\
95-115 \\
115-150 \\
\end{array}$ & $\begin{array}{l}\text { 10YR 4/3 } \\
\text { 10YR 5/3 } \\
\text { 10YR 6/3 } \\
\text { 10YR 6/3 } \\
\text { 10YR 6/3 } \\
\end{array}$ & $\begin{array}{l}3 / 3 \\
4 / 3 \\
4 / 3 \\
4 / 3 \\
4 / 3 \\
\end{array}$ & $\begin{array}{c}2 \mathrm{~m} \mathrm{sbk} \\
2 \mathrm{f} \mathrm{sbk} \\
2 \mathrm{~m} \mathrm{bk} \\
2 \mathrm{f} \mathrm{sbk} \\
2 \mathrm{c} \text { to f sbk }\end{array}$ & $\begin{array}{l}\text { v hard } \\
\text { ex hard } \\
\text { ex hard } \\
\text { hard } \\
s \text { hard }\end{array}$ & $\begin{array}{c}\text { firm } \\
\text { v firm } \\
\text { ex firm } \\
\text { friable } \\
\text { v friable }\end{array}$ & \begin{tabular}{|c} 
gradual s \\
gradual s \\
diffuse \\
diffuse \\
-
\end{tabular} \\
\hline & 9 & +9 & $\begin{array}{c}0-30 \\
30-50 \\
50-75 \\
75-95 \\
95-120 \\
\end{array}$ & $\begin{array}{l}\text { 10YR 5/3 } \\
\text { 10YR 5/3 } \\
\text { 10YR 6/3 } \\
\text { 10YR 6/3 } \\
\text { 10YR 6/3 } \\
\end{array}$ & $\begin{array}{l}4 / 3 \\
4 / 3 \\
4 / 3 \\
4 / 3 \\
4 / 3 \\
\end{array}$ & $\begin{array}{c}2 \mathrm{~m} \mathrm{sbk} \\
2 \mathrm{~m} \mathrm{bk} \\
2 \mathrm{~m} \mathrm{sbk} \\
1 \mathrm{f} \text { to } 2 \mathrm{~m} \mathrm{bk} \\
1 \mathrm{f} \text { to } 2 \mathrm{~m} \mathrm{gr}\end{array}$ & $\begin{array}{c}\text { ex hard } \\
\text { ex hard } \\
\text { ex hard } \\
\text { soft } \\
\text { soft } \\
\end{array}$ & $\begin{array}{c}\text { friable } \\
\text { v firm } \\
\text { ex firm } \\
\text { v friable } \\
\text { v friable }\end{array}$ & \begin{tabular}{|c} 
diffuse \\
gradual s \\
diffuse \\
diffuse \\
- \\
\end{tabular} \\
\hline & 10 & +8 & $\begin{array}{c}0-30 \\
30-60 \\
60-90 \\
90-120 \\
120-150 \\
\end{array}$ & $\begin{array}{l}\text { 10YR 5/3 } \\
\text { 10YR 5/3 } \\
\text { 10YR 5/3 } \\
\text { 10YR 6/3 } \\
\text { 10YR 6/3 } \\
\end{array}$ & $\begin{array}{l}4 / 3 \\
4 / 3 \\
4 / 3 \\
4 / 3 \\
4 / 3 \\
\end{array}$ & $\begin{array}{l}2 \mathrm{~m} \mathrm{bk} \\
2 \mathrm{~m} \mathrm{sbk} \\
2 \mathrm{~m} \mathrm{sbk} \\
2 \mathrm{~m} \mathrm{sbk} \\
2 \mathrm{~m} \mathrm{sbk} \\
\end{array}$ & $\begin{array}{l}\text { ex hard } \\
\text { ex hard } \\
\text { ex hard } \\
\text { hard } \\
\text { hard } \\
\end{array}$ & $\begin{array}{c}\text { ex firm } \\
\text { v firm } \\
\text { v firm } \\
\text { v friable } \\
\text { friable }\end{array}$ & $\begin{array}{c}\text { diffuse } \\
\text { diffuse } \\
\text { gradual s } \\
\text { diffuse } \\
- \\
\end{array}$ \\
\hline & 11 & +8 & $\begin{array}{c}0-30 \\
30-60 \\
60-90 \\
90-120 \\
120-150 \\
\end{array}$ & $\begin{array}{l}\text { 10YR 5/2 } \\
\text { 10YR 5/2 } \\
\text { 10YR 5/2 } \\
\text { 10YR 4/2 } \\
\text { 10YR 4/2 }\end{array}$ & $\begin{array}{l}4 / 2 \\
4 / 2 \\
4 / 2 \\
3 / 2 \\
3 / 2 \\
\end{array}$ & $\begin{array}{l}2 \mathrm{~m} \mathrm{bk} \\
2 \mathrm{~m} \mathrm{bk} \\
2 \mathrm{~m} \mathrm{bk} \\
2 \mathrm{~m} \mathrm{bk} \\
2 \mathrm{~m} \mathrm{bk}\end{array}$ & $\begin{array}{l}\text { ex hard } \\
\text { ex hard } \\
\text { ex hard } \\
\text { ex hard } \\
\text { ex hard }\end{array}$ & $\begin{array}{c}\text { firm } \\
\text { firm } \\
\text { firm } \\
\text { firm } \\
\text { ex firm }\end{array}$ & $\begin{array}{c}\text { diffuse } \\
\text { diffuse } \\
\text { gradual s } \\
\text { diffuse } \\
\text { - }\end{array}$ \\
\hline & 12 & +9 & $\begin{array}{c}0-30 \\
30-60 \\
60-90 \\
90-120 \\
120-150 \\
\end{array}$ & $\begin{array}{l}10 Y R \text { 5/3 } \\
\text { 10YR 5/3 } \\
\text { 10YR 5/3 } \\
\text { 10YR 5/3 } \\
\text { 10YR 4/3 }\end{array}$ & $\begin{array}{l}4 / 3 \\
3 / 3 \\
4 / 3 \\
3 / 3 \\
3 / 3 \\
\end{array}$ & $\begin{array}{c}2 \mathrm{~m} \mathrm{bk} \\
2 \mathrm{~m} \mathrm{sbk} \\
2 \mathrm{~m} \mathrm{bk} \\
2 \mathrm{~m} \mathrm{bk} \\
2 \mathrm{~m} \mathrm{bk}\end{array}$ & $\begin{array}{l}\text { ex hard } \\
\text { ex hard } \\
\text { ex hard } \\
\text { ex hard } \\
\text { ex hard }\end{array}$ & $\begin{array}{c}v \text { firm } \\
\text { firm } \\
\text { firm } \\
\text { firm } \\
\text { firm } \\
\end{array}$ & $\begin{array}{c}\text { diffuse } \\
\text { diffuse } \\
\text { diffuse } \\
\text { gradual s } \\
\text { - }\end{array}$ \\
\hline
\end{tabular}


M.S. Amira, et al.,

Table (2): Content.

\begin{tabular}{|c|c|c|c|c|c|c|c|c|c|}
\hline \multirow{2}{*}{\begin{tabular}{|c|} 
Geomor \\
phic \\
units \\
\end{tabular}} & \multirow{2}{*}{$\begin{array}{c}\text { Profile } \\
\text { No. }\end{array}$} & \multirow{2}{*}{$\begin{array}{c}\text { Elevation } \\
\text { m asl }\end{array}$} & \multirow{2}{*}{$\begin{array}{c}\text { Depth } \\
\text { cm }\end{array}$} & \multicolumn{2}{|c|}{ Color } & \multirow[b]{2}{*}{ Structure $^{1}$} & \multicolumn{2}{|c|}{ Consistence $^{2}$} & \multirow[b]{2}{*}{ Boundary ${ }^{3}$} \\
\hline & & & & Dry & Moist & & Dry & Moist & \\
\hline \multirow{5}{*}{ 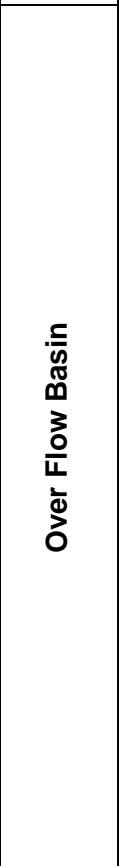 } & 13 & +8 & \begin{tabular}{|c|}
$0-30$ \\
$30-60$ \\
$60-90$ \\
$90-120$ \\
$120-140$ \\
\end{tabular} & $\begin{array}{l}\text { 10YR 4/3 } \\
\text { 10YR 5/3 } \\
\text { 10YR 4/3 } \\
\text { 10YR 4/3 } \\
\text { 10YR 4/3 }\end{array}$ & $\begin{array}{l}3 / 3 \\
3 / 3 \\
3 / 3 \\
3 / 3 \\
3 / 3 \\
\end{array}$ & $\begin{array}{l}2 \mathrm{mbk} \\
2 \mathrm{c} \mathrm{bk} \\
2 \mathrm{mbk} \\
2 \mathrm{mbk} \\
2 \mathrm{mbk}\end{array}$ & $\begin{array}{l}\text { ex hard } \\
\text { ex hard } \\
\text { ex hard } \\
\text { ex hard } \\
\text { ex hard }\end{array}$ & $\begin{array}{l}\text { ex firm } \\
\text { ex firm } \\
v \text { firm } \\
\text { ex firm } \\
\text { ex firm }\end{array}$ & $\begin{array}{l}\text { diffuse } \\
\text { diffuse } \\
\text { diffuse } \\
\text { diffuse } \\
-\end{array}$ \\
\hline & 14 & +8 & \begin{tabular}{|c|}
$0-30$ \\
$30-60$ \\
$60-90$ \\
$90-110$ \\
$110-130$ \\
$130-150$ \\
\end{tabular} & $\begin{array}{l}\text { 10YR 5/3 } \\
\text { 10YR 5/3 } \\
\text { 10YR 5/3 } \\
\text { 10YR 5/3 } \\
\text { 10YR 6/3 } \\
\text { 10YR 6/3 }\end{array}$ & $\begin{array}{l}3 / 3 \\
3 / 3 \\
3 / 3 \\
3 / 3 \\
4 / 3 \\
4 / 3 \\
\end{array}$ & $\begin{array}{l}2 \mathrm{~m} \mathrm{sbk} \\
2 \mathrm{~m} \mathrm{sbk} \\
2 \mathrm{~m} \mathrm{bk} \\
2 \mathrm{~m} \mathrm{sbk} \\
1 \mathrm{~m} \mathrm{sbk} \\
2 \mathrm{~m} \mathrm{sbk}\end{array}$ & $\begin{array}{c}\text { ex hard } \\
\text { ex hard } \\
\text { hard } \\
\text { hard } \\
\text { soft } \\
\text { hard } \\
\end{array}$ & $\begin{array}{c}\text { firm } \\
\text { firm } \\
\text { firm } \\
\text { firm } \\
\text { v friable } \\
\text { v firm } \\
\end{array}$ & $\begin{array}{c}\text { diffuse } \\
\text { diffuse } \\
\text { diffuse } \\
\text { diffuse } \\
\text { gradual s } \\
-\end{array}$ \\
\hline & 15 & +8 & \begin{tabular}{|c|}
$0-30$ \\
$30-60$ \\
$60-90$ \\
$90-120$ \\
$120-140$ \\
\end{tabular} & $\begin{array}{l}\text { 10YR } 5 / 3 \\
10 Y R 4 / 3 \\
10 Y R 4 / 3 \\
10 Y R 4 / 3 \\
10 Y R 4 / 3 \\
\end{array}$ & $\begin{array}{l}3 / 3 \\
3 / 3 \\
3 / 3 \\
3 / 3 \\
3 / 3 \\
\end{array}$ & $\begin{array}{l}2 \mathrm{mbk} \\
2 \mathrm{mbk} \\
2 \mathrm{~m} \mathrm{bk} \\
2 \mathrm{~m} \mathrm{bk} \\
2 \mathrm{c} \mathrm{bk} \\
\end{array}$ & $\begin{array}{l}\text { ex hard } \\
\text { ex hard } \\
\text { ex hard } \\
\text { ex hard } \\
\text { ex hard } \\
\end{array}$ & $\begin{array}{l}\text { friable } \\
\text { ex firm } \\
\text { ex firm } \\
\text { ex firm } \\
\text { ex firm } \\
\end{array}$ & 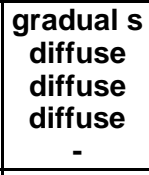 \\
\hline & 16 & +8 & $\begin{array}{c}0-30 \\
30-60 \\
60-90 \\
90-120 \\
120-150\end{array}$ & $\begin{array}{l}\text { 10YR 4/3 } \\
\text { 10YR 5/3 } \\
\text { 10YR 5/3 } \\
\text { 10YR 5/3 } \\
\text { 10YR 6/3 }\end{array}$ & $\begin{array}{l}3 / 3 \\
3 / 3 \\
3 / 3 \\
3 / 3 \\
4 / 3\end{array}$ & $\begin{array}{l}2 \mathrm{~m} \mathrm{sbk} \\
2 \mathrm{~m} \mathrm{bk} \\
2 \mathrm{~m} \mathrm{bk} \\
2 \mathrm{~m} \mathrm{bk} \\
2 \mathrm{~m} \mathrm{sbk}\end{array}$ & $\begin{array}{l}\text { ex hard } \\
\text { ex hard } \\
\text { ex hard } \\
\text { ex hard } \\
v \text { hard }\end{array}$ & $\begin{array}{c}\text { firm } \\
\text { ex firm } \\
\text { ex firm } \\
\text { firm } \\
\text { v firm }\end{array}$ & $\begin{array}{c}\text { gradual s } \\
\text { diffuse } \\
\text { diffuse } \\
\text { gradual s } \\
-\end{array}$ \\
\hline & 17 & +8 & \begin{tabular}{|c|}
$0-30$ \\
$30-60$ \\
$60-90$ \\
$90-120$ \\
$120-150$ \\
\end{tabular} & $\begin{array}{l}10 Y R 5 / 3 \\
10 Y R 6 / 3 \\
10 Y R 6 / 3 \\
10 Y R 6 / 3 \\
10 Y R 6 / 3 \\
\end{array}$ & $\begin{array}{l}3 / 3 \\
4 / 3 \\
4 / 3 \\
4 / 3 \\
4 / 3 \\
\end{array}$ & $\begin{array}{c}2 \mathrm{~m} \mathrm{bk} \\
2 \mathrm{~m} \mathrm{sbk} \\
1 \mathrm{f} \mathrm{sbk} \\
2 \mathrm{~m} \mathrm{sbk} \\
2 \mathrm{~m} \mathrm{sbk} \\
\end{array}$ & $\begin{array}{l}\text { v hard } \\
\text { hard } \\
\text { hard } \\
v \text { hard } \\
v \text { hard } \\
\end{array}$ & $\begin{array}{c}v \text { firm } \\
\text { friable } \\
\text { friable } \\
v \text { firm } \\
\text { friable } \\
\end{array}$ & \begin{tabular}{|c|}
$\begin{array}{c}\text { gradual s } \\
\text { gradual s } \\
\text { gradual s } \\
\text { diffuse } \\
-\end{array}$ \\
\end{tabular} \\
\hline 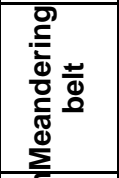 & 18 & +10 & $\begin{array}{c}0-30 \\
30-60 \\
60-90 \\
90-120 \\
120-150 \\
\end{array}$ & $\begin{array}{l}\text { 10YR 5/3 } \\
\text { 10YR 4/3 } \\
\text { 10YR 6/3 } \\
\text { 10YR 6/3 } \\
\text { 10YR 6/3 } \\
\end{array}$ & $\begin{array}{l}3 / 3 \\
3 / 3 \\
4 / 3 \\
4 / 3 \\
4 / 3 \\
\end{array}$ & $\begin{array}{c}2 \mathrm{~m} \text { sbk } \\
2 \mathrm{~m} \mathrm{sbk} \\
1 \mathrm{f} \mathrm{sbk} \text { to } \mathrm{gr} \\
1 \mathrm{f} \mathrm{gr} \\
1 \mathrm{f} \mathrm{gr} \\
\end{array}$ & $\begin{array}{c}\text { v hard } \\
\text { ex hard } \\
\text { soft } \\
\text { soft } \\
\text { soft } \\
\end{array}$ & $\begin{array}{c}\text { friable } \\
\text { firm } \\
\text { loose } \\
\text { loose } \\
\text { loose } \\
\end{array}$ & $\begin{array}{c}\text { gradual s } \\
\text { clear } \\
\text { diffuse } \\
\text { diffuse } \\
- \\
\end{array}$ \\
\hline 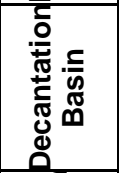 & 19 & +8 & \begin{tabular}{|c|}
$0-30$ \\
$30-60$ \\
$60-90$ \\
$90-120$ \\
$120-150$ \\
\end{tabular} & $\begin{array}{l}10 Y R 5 / 3 \\
10 Y \text { R } 5 / 3 \\
10 Y R 5 / 3 \\
10 Y R 4 / 3 \\
10 Y R 4 / 3 \\
\end{array}$ & $\begin{array}{l}3 / 3 \\
3 / 3 \\
3 / 3 \\
3 / 3 \\
3 / 3 \\
\end{array}$ & $\begin{array}{l}2 \mathrm{~m} \mathrm{bk} \\
2 \mathrm{~m} \mathrm{bk} \\
2 \mathrm{~m} \mathrm{bk} \\
2 \mathrm{~m} \mathrm{bk} \\
2 \mathrm{~m} \mathrm{bk} \\
\end{array}$ & $\begin{array}{l}\text { ex hard } \\
\text { ex hard } \\
\text { ex hard } \\
\text { ex hard } \\
\text { ex hard } \\
\end{array}$ & $\begin{array}{l}\text { ex firm } \\
\text { ex firm } \\
\text { ex firm } \\
\text { ex firm } \\
\text { ex firm }\end{array}$ & $\begin{array}{l}\text { diffuse } \\
\text { diffuse } \\
\text { diffuse } \\
\text { diffuse } \\
-\end{array}$ \\
\hline $\begin{array}{l}\frac{5}{0} \\
\frac{0}{0} \\
\frac{d}{0} \\
\frac{0}{0}\end{array}$ & 20 & +8 & \begin{tabular}{|c|}
$0-30$ \\
$30-60$ \\
$60-90$ \\
$90-120$ \\
$120-150$ \\
\end{tabular} & $\begin{array}{l}\text { 10YR 4/3 } \\
\text { 10YR 4/3 } \\
\text { 10YR 4/3 } \\
\text { 10YR 4/3 } \\
\text { 10YR 4/3 }\end{array}$ & $\begin{array}{l}3 / 3 \\
3 / 3 \\
3 / 3 \\
3 / 3 \\
3 / 3\end{array}$ & $\begin{array}{l}2 \mathrm{~m} \text { to c sbk } \\
2 \mathrm{~m} \text { to } \mathrm{c} \mathrm{bk} \\
2 \mathrm{c} \text { to } \mathrm{m} \mathrm{bk} \\
2 \mathrm{c} \text { to } \mathrm{m} \mathrm{bk} \\
2 \mathrm{~m} \mathrm{bk}\end{array}$ & $\begin{array}{c}\text { ex hard } \\
\text { ex hard } \\
\text { ex hard } \\
\text { ex hard } \\
\text { hard }\end{array}$ & $\begin{array}{c}\text { firm } \\
\text { firm } \\
\text { ex firm } \\
\text { ex firm } \\
\text { ex firm }\end{array}$ & $\begin{array}{l}\text { diffuse } \\
\text { diffuse } \\
\text { diffuse } \\
\text { diffuse } \\
-\end{array}$ \\
\hline \multirow{3}{*}{ ฏ } & 21 & +9 & $\begin{array}{c}0-30 \\
30-70 \\
70-85 \\
85-120 \\
120-150\end{array}$ & $\begin{array}{l}10 Y R 4 / 3 \\
10 Y R 4 / 3 \\
10 Y R 5 / 3 \\
10 Y R 5 / 3 \\
10 Y R 5 / 3\end{array}$ & $\begin{array}{l}3 / 3 \\
3 / 3 \\
3 / 3 \\
3 / 3 \\
3 / 3 \\
\end{array}$ & $\begin{array}{c}2 \mathrm{~m} \mathrm{bk} \\
2 \mathrm{~m} \mathrm{sbk} \\
1 \mathrm{~m}, \mathrm{f} \mathrm{sbk} \\
1 \mathrm{f}, \mathrm{m} \mathrm{sbk} \\
2 \mathrm{~m} \mathrm{sbk}\end{array}$ & $\begin{array}{l}\text { ex hard } \\
\text { ex hard } \\
\text { hard } \\
v \text { hard } \\
\text { ex hard }\end{array}$ & $\begin{array}{l}v \text { firm } \\
v \text { firm } \\
\text { friable } \\
\text { firm } \\
\text { firm }\end{array}$ & $\begin{array}{c}\text { diffuse } \\
\text { clear } \\
\text { diffuse } \\
\text { clear } \\
-\end{array}$ \\
\hline & 22 & +8 & $\begin{array}{c}0-30 \\
30-60 \\
60-90 \\
90-130\end{array}$ & $\begin{array}{l}10 Y R 5 / 3 \\
10 Y R 6 / 3 \\
10 Y R 5 / 3 \\
10 Y R 5 / 3\end{array}$ & $\begin{array}{l}3 / 3 \\
4 / 3 \\
3 / 3 \\
3 / 3\end{array}$ & $\begin{array}{c}2 \mathrm{~m} \mathrm{sbk} \\
1 \mathrm{~m} \mathrm{f}, \mathrm{sbk} \\
1 \mathrm{~m} \mathrm{f}, \mathrm{sbk} \\
1 \mathrm{fgr}\end{array}$ & $\begin{array}{c}\text { ex hard } \\
\text { hard } \\
\text { soft } \\
\text { soft }\end{array}$ & $\begin{array}{c}\text { friable } \\
\text { friable } \\
\text { v friable } \\
\text { friable }\end{array}$ & $\begin{array}{c}\text { gradual s } \\
\text { gradual s } \\
\text { gradual s } \\
-\end{array}$ \\
\hline & 23 & +7 & $\begin{array}{c}0-30 \\
30-70 \\
70-90 \\
90-110 \\
110-150\end{array}$ & $\begin{array}{l}10 Y R \text { 5/3 } \\
\text { 10YR 6/3 } \\
10 \text { YR 6/3 } \\
10 Y R 6 / 3 \\
10 Y R \text { 5/3 }\end{array}$ & $\begin{array}{l}4 / 3 \\
4 / 3 \\
4 / 3 \\
4 / 3 \\
3 / 3\end{array}$ & $\begin{array}{c}1 \mathrm{~m}, \mathrm{f} \mathrm{sbk} \\
1 \mathrm{~m}, \mathrm{f} \mathrm{sbk} \\
1 \mathrm{~m}, \mathrm{f} \mathrm{sbk} \\
1 \mathrm{~m}, \mathrm{f} \mathrm{sbk} \\
2 \mathrm{~m} \text { sbk }\end{array}$ & $\begin{array}{l}\text { hard } \\
\text { hard } \\
\text { hard } \\
\text { hard } \\
\text { hard }\end{array}$ & $\begin{array}{c}\text { friable } \\
\text { friable } \\
\text { friable } \\
\text { friable } \\
\text { v friable }\end{array}$ & $\begin{array}{c}\text { gradual s } \\
\text { diffuse } \\
\text { diffuse } \\
\text { clear } \\
-\end{array}$ \\
\hline$\frac{\bar{\sigma}}{\frac{\pi}{0}}$ & 24 & +8 & $\begin{array}{c}0-30 \\
30-60 \\
60-90 \\
90-120 \\
120-150\end{array}$ & $\begin{array}{l}10 Y R \text { 5/3 } \\
10 Y R 5 / 3 \\
10 Y R 5 / 4 \\
10 Y R \text { 6/3 } \\
10 Y R 5 / 3\end{array}$ & $\begin{array}{l}3 / 3 \\
3 / 3 \\
4 / 3 \\
4 / 3 \\
4 / 3\end{array}$ & $\begin{array}{c}2 \mathrm{c}, \mathrm{m} \mathrm{bk} \\
2 \mathrm{c}, \mathrm{m} \text { bk } \\
2 \mathrm{~m}, \mathrm{f} \mathrm{sbk} \\
2 \mathrm{~m}, \mathrm{f} \mathrm{bk} \\
2 \mathrm{~m}, \mathrm{f} \mathrm{sbk}\end{array}$ & $\begin{array}{c}\text { ex hard } \\
\text { ex hard } \\
\text { hard } \\
\text { ex hard } \\
\text { hard }\end{array}$ & $\begin{array}{c}\text { firm } \\
\text { firm } \\
\text { friable } \\
\text { firm } \\
\text { friable }\end{array}$ & $\begin{array}{c}\text { diffuse } \\
\text { gradual s } \\
\text { gradual s } \\
\text { gradual s } \\
-\end{array}$ \\
\hline
\end{tabular}

Abbreviations: Texture*: L=loamy, $\mathrm{S}=$ sandy, $\mathbf{s}$ g=slightly gravely, g=gravely; Structure ${ }^{1}$ : 1=weak, 2 = moderate,$v=$ very, $\mathrm{f}=$ fine, $\mathrm{m}=$ medium, $\mathrm{co}=$ coarse, $\mathrm{gr}=$ granular, $\mathrm{sbk}=$ subangular blocky; Consistence ${ }^{2}$ : $s=$ slightly, $v=$ very, $x=$ extremely; Boundary ${ }^{3}$ s= smooth 


\section{Physiochemical Properties}

The results in Table (3) show that, these soils have mostly loam to sandy clay loam texture (as w.p.m.). They are non-saline as indicated by their EC values (< $1 \mathrm{dSm}^{-1}$, (wpm). Soil reaction is moderately alkaline as the $\mathrm{pH}$ values are between 7.5 to 8.3 .

These soils are slightly calcareous as shown from their $\mathrm{CaCO}_{3}$ contents that are mostly < 3.0 \% (as w.p.m.). Gypsum content is very low (<1\%). Organic matter (OM) is low (<20.0 g/kg, as w.p.m.) and decreased with depth. The cation exchange capacity (CEC) is depending on the fine fractions and organic matter contents. ESP values are lower than $15 \%$ indicating non sodicity effect in all studied soils.

\section{Soil Classification.}

The studied soils were classified up to sub great group level according to Soil Survey Staff (2014). The dominant soil moisture regime is "Torric" with
"Thermic" soil temperature regime. All studied soils haven't any diagnostic subsurface horizons. Therefore, these soils were classified under Entisols mostly as Typic Torriorthents.

\section{Land Capability Evaluation}

The Applied System of Land Evaluation (ASLE) model developed by Ismail et al. (2005) was used to assess the land capability for the studied soils. The land capability indices for these soils were obtained from the integration between this model and ArcGIS software based on the soil physical and chemical characteristics. The final land capability indices and classes for the soils of the studied area are presented in Table (4). Also, the spatial land capability classes map for this area are illustrated in Fig (3). Table (5) shows the areas of land capability classes for the studied soils. Results indicated that, about $48 \%$ of the studied soils have a Good (C2) capability class and the rest are considered as a Fair (C3) one. 
M.S. Amira, et al.,

Table (3): Some physical and chemical properties of studied soil profiles.

\begin{tabular}{|c|c|c|c|c|c|c|c|c|c|c|c|c|c|}
\hline \multirow{2}{*}{ 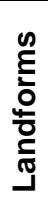 } & \multirow{2}{*}{$\begin{array}{l}\frac{0}{Z} \\
\frac{0}{c} \\
\frac{0}{0} \\
\frac{0}{0}\end{array}$} & \multirow[b]{2}{*}{$\begin{array}{l}\text { Depth } \\
\text { Cm }\end{array}$} & \multicolumn{3}{|c|}{$\begin{array}{c}\text { Particle size } \\
\text { distribution \% }\end{array}$} & \multirow{2}{*}{ 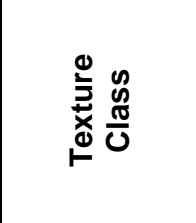 } & \multirow{2}{*}{ 돌 } & \multirow{2}{*}{ ש } & \multirow{2}{*}{ 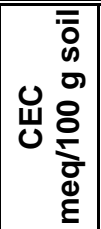 } & \multirow[b]{2}{*}{ ESP } & \multirow{2}{*}{ 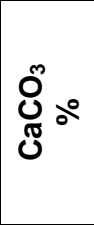 } & \multirow{2}{*}{$\sum_{0}^{\frac{5}{2}} \overbrace{}^{0}$} & \multirow{2}{*}{$\begin{array}{l}\text { OM } \\
\text { g/kg }\end{array}$} \\
\hline & & & ஸे & 紊 & $\frac{\widehat{\pi}}{0}$ & & & & & & & & \\
\hline \multirow{24}{*}{ 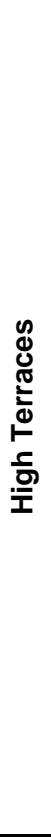 } & \multirow{6}{*}{1} & $0-30$ & $\varepsilon \varepsilon, Y \mu$ & $r y, 1$ & . & Clay Loam & $V, q Y$ & • & $r_{0, \varepsilon .}$ & $1 \cdot, r 0$ & 4,94 & $\cdot, 11$ & 10,1 . \\
\hline & & $30-60$ & 1,10 & $r_{0,1}$ & $r q, .0$ & Sandy clay L & $V, 94$ & • & $r r, r$. & $11,1 r$ & $r, r_{\Lambda}$ & $\cdot, 10$ & $v, r$. \\
\hline & & $60-90$ & $9, \mathrm{~V}_{0}$ & r४,O & $r \mu, v_{0}$ & Sandy clay L & $\Lambda, .0$ & • r r & $r_{\bullet}, r_{\text {. }}$ & $11, \varepsilon \leqslant$ & $r, Y$. & $\cdot, 14$ & $\wedge, \varepsilon$. \\
\hline & & $90-110$ & $\Delta r, r$. & $r q, v$ & $11, \ldots$ & Loam & 1,11 & $\cdot r_{1}$ & $10,1$. & $I r, I V$ & $1, r V$ & $\cdot, 17$ & $\Lambda, \varepsilon$. \\
\hline & & 110 & $0 \cdot, \cdot r$ & 40.5 & 9.47 & Loam & 8.14 & 0.32 & 9.30 & 13.221 & 1.29 & 0.19 & 8.40 \\
\hline & & W.P.M & 48.63 & 29.72 & 21.65 & Loam & 8.03 & 0.33 & 18.84 & 11.60 & 2.58 & 0.15 & 9.68 \\
\hline & & $0-30$ & $\varepsilon r, q$. & $r \leqslant, 0$. & $r 1, \pi$. & Clay Loam & $V, T \pi$ & $\cdot, r$. & $r q, q$. & $9, \wedge 0$ & ז, ז & $\cdot, 1 \varepsilon$ & $11, \Lambda$. \\
\hline & & & $\varepsilon \leqslant, 0$. & $r_{0}, 1$. & r., \&. & Sandy clay L & $V, 94$ & $\cdot, r_{1}$ & $r_{0}, q$. & $1,, r_{0}$ & $r, T \leqslant$ & . זr & $1 ., 9$. \\
\hline & & $60-90$ & $\varepsilon \wedge, 0$. & $r V, I r$ & $r \varepsilon, r \Lambda$ & Loam & $\Lambda, 1 \leqslant$ & $\cdot, Y^{\prime}$ & $1 \wedge, \varepsilon$. & $1 \cdot, r_{0}$ & $r, Y \cdot Y$ & $\cdot, 17$ & $v, r$. \\
\hline & 2 & $90-115$ & $0 ., 9$. & r., & $1 \Lambda, V_{0}$ & Loam & $\Lambda, Y Y$ & $\cdot, r$. & $1 \%, q$. & $|r, 1|$ & r, 94 & - r & $Y, Y$. \\
\hline & & & $\varepsilon q, q$. & 31.48 & 18.62 & Loam & 8.30 & $\cdot, r$. & 10.20 & 12.5 & 1.013 & 0.11 & 3.40 \\
\hline & & W.P.M & $\leqslant v, 01$ & 27.74 & 24.75 & Sandy Clay L & 8.05 & $\cdot, r_{1}$ & 19.54 & 11.01 & 2.53 & 0.17 & 7.82 \\
\hline & & $0-30$ & $\leq \varepsilon, 19$ & $r, Y$. & $r \varepsilon, \pi$ & Clay loam & $\Lambda, Y \wedge$ & $\cdot r \mu$ & $r \cdot, \varepsilon$. & $\mid r, 1 \leq$ & $r, r$. & $\cdot, 11$ & $1 \wedge, \varepsilon \cdot$ \\
\hline & & & 1. & rY,O. & $r r, \varepsilon$. & Clay I & $\Lambda, r_{1}$ & $\cdot, \leqslant 0$ & $\Lambda$, Or & $1 \%, 10$ & o & $\cdot, \mathrm{IV}$ & $11,0$. \\
\hline & & $60-90$ & $v, \infty$ & ro,v. & $r q, v_{0}$ & Sandy clay L & $\Lambda, 10$ & $\cdot, \varepsilon \wedge$ & $r \cdot, 1$. & $I \mu, \Lambda$. & $r, r q$ & $\cdot, 1 \leqslant$ & $\wedge, \ldots$ \\
\hline & 3 & $90-$ & $01, Y$. & $r \cdot, r \mu$ & $1 \Lambda, \leqslant V$ & Loam & $\Lambda, r v$ & $\cdot, \leqslant 1$ & $10, \mathrm{~V} \mid$ & $I \leqslant, Y Y$ & $1, v \leqslant$ & $\cdot, 1 \mathrm{r}$ & $\neg, \ldots$ \\
\hline & & & $\varepsilon \Lambda, 1 r$ & 33.17 & 18.70 & Loam & $\Lambda, r_{1}$ & $\cdot, r_{0}$ & $11,9$. & $\mid \leq, V 01$ & $1, \pi V$ & $\cdot, 10$ & $\varepsilon, 0$. \\
\hline & & W.F & $\varepsilon V, Y \mu$ & 26.58 & 26.19 & Sandy Clay L & 8.24 & $\cdot, r q$ & 21.33 & $|r, 9|$ & $r, \varepsilon \wedge$ & $\cdot, 1 \leqslant$ & 9,71 \\
\hline & & $0-$ & $\varepsilon \cdot$, & YY,YY & $r v, \leqslant \Lambda$ & Clay loam & $V, 99$ & $\cdot, 04$ & $r q, 1$. & IY,YO & $r, 11$ & $\cdot, 19$ & $T V, V$. \\
\hline & & &,$\cdot 1$ & ro,v. & $r, Y, r$ & Clay loam & $\Lambda, r$. & $\cdot$, Or & $Y_{0}, q$. & $1 r, 70$ & $r, r V$ & $\cdot, I V$ & $1+0$. \\
\hline & 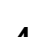 & & , vo & $r v, 11$ & $Y_{0}, 1 \leqslant$ & Sandy clay L & $\Lambda, \varepsilon$. & $\cdot, 0$. & $r \cdot, v$. & $1 r, 1$. & $r, \varepsilon$. & $\cdot, r_{1}$ & $\wedge, \ldots$ \\
\hline & 4 & & $\varepsilon q, .0$ & $r \cdot, \mu_{0}$ & $r_{\cdot}, \tau_{.}$ & Loa & $\Lambda, Y \wedge$ & $\cdot, 0$. & $17, r$. & Ir,YY & $1, \mathrm{rN}$ & $\cdot, 19$ & $v, \ldots$ \\
\hline & & 120 & $\{0,10$ & 31.50 & 23.35 & Loa & 8.18 & 0.44 & 15.60 & 13.80 & 1.65 & 0.12 & 6.00 \\
\hline & & W.F & $\{0, .7$ & 26.91 & 28.03 & Sandy & 8.24 & 0.51 & 21.51 & 12.92 & 2.24 & 0.18 & 10.93 \\
\hline & & & $\varepsilon \varepsilon, \varepsilon r$ & $r \cdot, r$. & $r \Delta, r \Lambda$ & Loam & $v, \Lambda$. & $\cdot, \leqslant 4$ & $r v, v$. & $M, I r$ & $\varepsilon, 7 r$ & $\cdot, 1 \mathrm{~V}$ & $r 1.00$ \\
\hline & & &,$\ldots$ & $r r, \Lambda$. & $r \cdot, r$. & ay L & $v, \Lambda 1$ & • & Y 0,7 . & $\mid r, 01$ & צוד, & •, Yr & $1 \wedge, 0$ \\
\hline & & $60-90$ & $\varepsilon q, 1 \vee$ & $r_{0}, q$. & $r \varepsilon, q r$ & Sandy clay L & $\vee, q q$ & . , $\{$. & $\varphi_{\cdot}, \ldots$. & $1 r, 1$. & $\uparrow, १ \wedge$ & $\cdot, r_{1}$ & $v, 0$. \\
\hline & $\mathbf{b}$ & $90-120$ & $0 \leqslant, \Lambda$. & $r \Lambda, \Lambda$. & $17, \varepsilon$. & Sandy Loam & $\Lambda, Y Y$ & •,$\varepsilon r$ & $1 \varepsilon, Y$. & $1 r, 1$. & $r, r$. & $\cdot, 1 \mu$ & $\checkmark, r$. \\
\hline & & $120-150$ & $0 ., 10$ & rq, 0. & 19,10 & Loam & $\Lambda, r_{0}$ & $\cdot, 0 \leqslant$ & $I V, v$. & $\mid r, 0$ & 1.67 & 0.15 & 5.00 \\
\hline & & W.P.M & $\leq q, Y)$ & Y & $r_{0}, r_{0}$ & Sandy Clay L & $\Lambda, \cdot r$ & $\cdot, \leqslant 7$ & $r, 1 r$ & $\mid r, 71$ & 3.05 & 0.18 & 11.64 \\
\hline ఫ్ర & & $0-30$ & $\varepsilon Y, 0$. & YY,O. & $r_{0, \ldots}$ & Clay Loam & $V, 90$ & $\cdot, \leqslant \leqslant$ & $r q, v$. & $1 \cdot, r 0$ & $\varepsilon, 00$ & $\cdot, 1 Y$ & $r_{\cdot}, r_{\cdot}$ \\
\hline & & &,$\wedge$ & $\theta, r o$ & $r q, \Lambda$. & Clay & $\Lambda, 1$. & שr & $r o, v$. & $1 \cdot$, ro & $r, \leqslant V$ & $\cdot, 19$ & $9, \wedge$. \\
\hline & 6 & $60-90$ & $\varepsilon \uparrow, \ldots$ & $r v, 11$ & $P ४, \wedge q$ & Sandy clay L & $\wedge, 1$. & ש & $r \mu, r$. & $1 ., 9$. & 1,41 & $\cdot, I V$ & $\wedge .00$ \\
\hline & 6 & $90-120$ & $\varepsilon 9,7 \varphi$ & $r \wedge, \ldots$ & $r Y, r v$ & Loam & $\Lambda, \cdot 9$ & • r rr & $r_{\cdot}, \varepsilon$. & $11,1$. & $1, r \Lambda$ & $\cdot, 10$ & $v, 0$. \\
\hline & & & $\varepsilon 0, \ldots$ & $r \cdot, 1 \varepsilon$ & $Y \leqslant, \wedge \uparrow$ & Loam & $\Lambda, 1 \leqslant$ & $\cdot, \mu 1$ & $11,0$. & $\mid r, 0,1$ & $\varepsilon, \infty$ & $\cdot, 1 \mathrm{r}$ & $\varepsilon, \wedge$. \\
\hline & & & $\leqslant 0,7$. & rq, Tr & $r V, V \Lambda$ & Sandy Clay L & $\Lambda, \cdot \Lambda$ & . r. & $r M, O r$ & 11,11 & $r, q r$ &., 10 & $1 \cdot, \cdot 4$ \\
\hline & & $0-30$ & $r 7,11$ & $r_{0, \ldots}$ & $r \wedge, \wedge r$ & Clay Loam & $v, v q$ & $\cdot, \leqslant \wedge$ & $r \theta, v$. & 14,11 & $r, \wedge 0$ &., 19 & $19, \pi \cdot$ \\
\hline & & & &,$\wedge \wedge$ & i, OY & & , & $\cdot, \leqslant 0$ & $r_{\cdot}, 0$. & r, vo & $r, T \varepsilon$ & $\cdot, 11$ & $\wedge, \bullet$. \\
\hline & 7 & & &,$\wedge \Lambda$ & $7, \leqslant \leqslant$ & & $\vee, q \vee$ & $\cdot, \leqslant 9$ & $\vee, \uparrow$. & I & $r, r v$ & $\cdot, 19$ & $V, r$. \\
\hline & I & & & $r \Lambda, 1 \mu$ & $1 \cdot, V_{4}$ & Loam & $v, q 0$ & $\cdot, O Y$ & $10, \pi$. & $1 \leqslant, 11$ & $1, \pi 4$ & $\cdot, I V$ & 7.00 \\
\hline & & & o & $r v, \ldots$ & $v, r_{0}$ & Sandy Loam & $\wedge, 1$. & • & 14.10 & $\mid 1,701$ & $1, \cdot 9$ & $\cdot, r \mu$ & 0,1 \\
\hline & & & $\varepsilon 0,1 \wedge$ & $r \wedge, r \Lambda$ & $17, \leqslant \leq$ & & $v, q r$ & ד & 18.64 & $1 \Gamma, \Lambda_{0}$ & $r, . r$ & $\cdot, I V$ & $q, r q$ \\
\hline & & & $r q, 1$. & $r \cdot, .$. & $r_{\cdot, q}$, & $\mathrm{Cla}$ & $\vee, \wedge \downarrow$ & $\cdot, r_{1}$ & $r Q, V$. & 11,11 & $\varepsilon, 0 \wedge$ & $\cdot, 1 Y$ & $19,0$. \\
\hline & & & T, r Y & & $r v,{ }_{T}$ & lay Loam & $\vee, \wedge \Lambda$ & $\cdot, r_{0}$ & $r \mu, \Lambda$. & $11,0$. & $\Gamma, v \varepsilon$ & $\cdot, 1 \leqslant$ & $9, \ldots$ \\
\hline & 8 & $70-95$ & $\varepsilon 4, \uparrow \vee$ & $r r, \ldots$ & $r, \mu r$ & Loam & $V, \wedge r$ & • & $19,8$. & $11,0 \mathrm{~V}$ & ५,9 & $\cdot, I V$ & $\wedge, \varepsilon$. \\
\hline & $\mathbf{0}$ & $95-115$ & $\varepsilon \wedge, \ldots$ & $r r, v$. & $11, r$ & Loam & $v, \Lambda$. & - , Y O & $1 \mu, \ldots$ & $\mid r, Y 4$ & $\uparrow, \uparrow \wedge$ & $\cdot, 1 \mathrm{r}$ & 7,1 . \\
\hline ن্ّ & & $115-150$ & 47.50 & 33.50 & 19.00 & Loam & 7.93 & 0.20 & 10.20 & 12.66 & 1.68 & $\cdot, 1 r$ & 5.50 \\
\hline & & W.P.M & 44.37 & 31.64 & 23.99 & Loam & 7.87 & 0.25 & 18.88 & 11.81 & 3.32 &., $1 \mathrm{~T}$ & 9.80 \\
\hline 5 & & $0-30$ & TV, & $10,1$. & $\leqslant V, \cdot \varepsilon$ & Clay & $v, \wedge_{0}$ & $\cdot, Y q$ & $r \cdot, \ldots$ & 11,10 & 4,97 & $\cdot, 19$ & $r_{0,7}$ \\
\hline 3. & & & $\leqslant \varphi, 0$. & IV,o. & $\varepsilon, \ldots$ & Clay & V,q & • & $r \Lambda, 1$. & 1. r. & r, & $\cdot, 10$ & $11, \wedge$. \\
\hline & 9 & $50-75$ & $\varepsilon \varepsilon, \wedge \vee$ & $r 0,1 r$ & $\mu_{\cdot}, \ldots$ & Clay Loam & $\vee, \wedge \Lambda$ & • & ro,r. & $11,0$. & $1, r Y$ & דוץ & $\Lambda, \varepsilon$. \\
\hline & 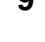 & & or, $\{$. & $r \cdot, 1 \wedge$ & $r v, \leqslant r$ & Sandy clay L & $v, 7)$ & $\cdot, r \mu$ & $r v, 0$. & 11,70 & - ,OYA & $\cdot, 11$ & $0, \ldots$ \\
\hline & & & & 18.35 & 18.52 & Sandy loam & 7.71 & 0.35 & 17.90 & 12.13 & 0.32 & 0.13 & 6.70 \\
\hline & & W.P.M & $\varepsilon \vee, \vee \wedge$ & 19.11 & 33.11 & Sandy Clay L & 7.80 & 0.32 & 25.77 & 11.54 & 2.02 & 0.17 & 12.35 \\
\hline
\end{tabular}

W.P.M = weighted profile means, L= loam 
A recent geomorphic and pedological studies on Menouf province soils,

Table (3): Cont.

\begin{tabular}{|c|c|c|c|c|c|c|c|c|c|c|c|c|c|}
\hline \multirow{2}{*}{ 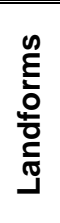 } & \multirow{2}{*}{ 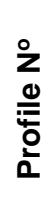 } & \multirow{2}{*}{$\begin{array}{c}\text { Depth } \\
\text { cm }\end{array}$} & \multicolumn{3}{|c|}{$\begin{array}{c}\text { Particle size } \\
\text { distribution \% }\end{array}$} & \multirow{2}{*}{ 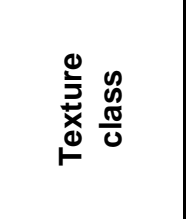 } & \multirow{2}{*}{ 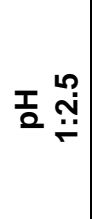 } & \multirow{2}{*}{ ن } & \multirow{2}{*}{ 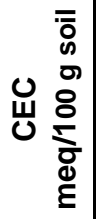 } & \multirow[b]{2}{*}{ ESP } & \multirow{2}{*}{$\bigcup_{0}^{\infty} \overbrace{0}^{\circ}$} & \multirow{2}{*}{$\sum_{0}^{\frac{E}{0}} \overbrace{0}^{\circ}$} & \multirow[b]{2}{*}{$\begin{array}{l}\text { OM } \\
\text { g/kg }\end{array}$} \\
\hline & & & 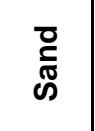 & $\frac{ \pm}{\bar{n}}$ & $\frac{\vec{\sigma}}{U}$ & & & & & & & & \\
\hline \multirow{18}{*}{ 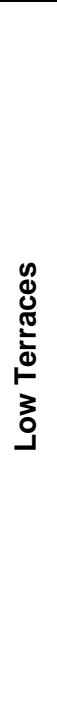 } & \multirow{6}{*}{10} & $0-30$ & $\leqslant \varepsilon, 90$ & $r \cdot, r$. & $r \leqslant, V 0$ & Loam & $v, q \leq$ & $\cdot, Y \Lambda$ & Yo,, . & 11,11 & $r, Y \leq$ & $\cdot, 10$ & Y1, \&. \\
\hline & & $30-60$ & $\leq \vee, \Lambda$. & $r \varepsilon, 1 r$ & $1 \Lambda, \cdot V$ & Loam & $\Lambda, \cdot r$ & $\cdot, Y \wedge$ & $r Y, Y$. & $\mid r, \varepsilon$. & $r, q \vee$ & $\cdot, 1 \leq$ & $10, \ldots$ \\
\hline & & $60-90$ & $\leq 9,9 \wedge$ & $r q, 0$. & $1,0 Y$ & Loam & $\Lambda, r Y$ & • , Yo & $17, r$. & Ir,qY & $r, \leqslant 9 \wedge$ & $\cdot, 1 r$ & $\Lambda, r$. \\
\hline & & $90-120$ & $01,1$. & $\varepsilon \cdot, \varepsilon$ & $\wedge, 0$ & Loam & $\Lambda, \cdot r$ & $\cdot, r \cdot$ & $\mid r, \ldots$ & ס ט, & $r, r v q$ & • , rr & $v, r$. \\
\hline & & $120-150$ & $01, Y 1$ & 37.11 & 11.68 & Loam & 8.31 & $\cdot, Y_{1}$ & $1 \cdot, 7$ & $\mid r, T$ & 1,70 &., 10 & $0, r$. \\
\hline & & W.P.M & $\{9, .1$ & 36.29 & 36.29 & Loam & 8.10 & $\cdot, r_{0}$ & 17.54 & $1 r, \wedge q$ & $r, \varepsilon r$ & $\cdot, 17$ & $11, \varepsilon r$ \\
\hline & \multirow{6}{*}{11} & $0-30$ & $r \leqslant, 70$ & $r \cdot, r$. & $\{0,10$ & Clay & $V, q v$ & $\cdot, \leqslant$. & $r \leqslant, 0$. & $M, 1 r$ & $r$ & $\cdot, 1 \leq$ & $Y \varepsilon, \varepsilon$ \\
\hline & & $30-60$ & $r v, r \leqslant$ & $r_{\cdot}, 1$. & $\{Y, 0 Y$ & Clay & $v, q v$ & $\cdot, 01$ & $r_{1}, 0$. & Ir, $\{$. & 1,91 & $\cdot, 11$ & 17,0 \\
\hline & & $60-90$ & $\leq 1, v$. & $r_{0, \varepsilon}$. & rr,q. & Clay Loam & $\Lambda, 10$ & $\cdot, \pi$ & $r v, 0$. & IT,YY & $1, r_{1}$ & $\cdot, 1 r$ & $v, 1$. \\
\hline & & $90-120$ & $\varepsilon r, \uparrow$. & $r V, r$. & $r q, Y$. & Clay Loam & $\Lambda, 1 \leq$ & $\cdot, 0$ & $r r, r$. & $14, \leqslant 0$ & $1, r \wedge 4$ & $\cdot, 19$ & $\checkmark, \wedge$ \\
\hline & & $120-150$ & $r q, \varepsilon r$ & 33.40 & 27.18 & Clay Loam & 8.25 & 0.57 & 20.20 & 13.65 & 0.726 & 0.16 & 5.00 \\
\hline & & W.P.M & $r q, r \leq$ & 25.26 & 35.40 & Clay Loam & 8.10 & 0.52 & 27.38 & 12.97 & 1.81 & 0.15 & 11.96 \\
\hline & \multirow{6}{*}{12} & $0-30$ & $\varepsilon \cdot \wedge r$ & $r Y, O$. & rq, 71 & Clay Loam & $\Lambda, r v$ & $\cdot, \leqslant Y$ & $r \wedge, \varepsilon$. & $\mid r, 11$ & $r, v \cdot r$ & $\cdot, 19$ & $r \leqslant, r$ \\
\hline & & $30-60$ & $\leq \neg, r \wedge$ & $r_{0, \varepsilon .}$ & $r \wedge, Y r$ & Sandy clay L & $\Lambda, r r$ & $\cdot, \infty$ & ro,, . & $\mid r, \leqslant 0$ & $1,91 \leq$ & $\cdot, 17$ & 10,9 \\
\hline & & $60-90$ & $0 ., r \cdot$ & ro,q. & $r r, q$. & Sandy clay L & $\Lambda, r Y$ & צדו, & $r_{\bullet}, \varepsilon$. & $1 r, 90$ & ד & $\cdot, 1 \leq$ & $q, r$. \\
\hline & & $90-120$ & $0 \leqslant, r$. & $r \Lambda, 1 \leqslant$ & IV, ד & Sandy Loam & $\Lambda, r \varepsilon$ & $\cdot, V^{\prime}$ & $1 \wedge, \ldots$ & or, & 1, & •, rr & $v, r$. \\
\hline & & $120-150$ & $\varepsilon \wedge, \varepsilon \cdot$ & $r \cdot, r$. & $r, r$. & Loam & $\Lambda, r$. & rז, & $1 r, \ldots$ & $1 r, q$. & 0.924 & 0.11 & 4.10 \\
\hline & & W.P.M & $\leq \wedge, \cdots$ & $Y 4, \leqslant 0$ & $r 0,00$ & Sandy Clay L & $\Lambda, r q$ & $\cdot, 7$ & M $1, \Lambda$. & $1 \%, 90$ & 1.66 & 0.16 & 12.16 \\
\hline \multirow{31}{*}{ 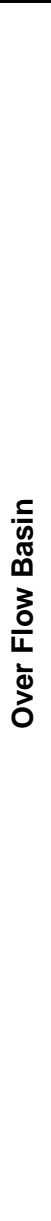 } & & $0-30$ & $r \leqslant, \Lambda$. & $r \cdot, \ldots$ & $r_{0, Y .}$ & Clay Loam & $\vee, \wedge V$ & $\cdot, 99$ & YY, T. & 9,10 & $r, \wedge \uparrow \leq$ & $\cdot, 10$ & $10,7$. \\
\hline & & $30-60$ & $\{1,19$ & $r r, 1$. & $r q, V I$ & Clay Loam & $\Lambda, .0$ & $\cdot, 9 \leq$ & $r_{\bullet}, \ldots$ & $9,0$. & $\curlyvee, \uparrow \wedge \uparrow$ & $\cdot, 11$ & $1 \cdot, 0$. \\
\hline & & $60-90$ & $\leq 9,79$ & $r_{0, \Lambda}$ & $1 \leq, \leq 7$ & Loam & $\Lambda, \cdot \varepsilon$ & $\cdot, \mathrm{VV}$ & $\Lambda \Lambda, Y$. & $11, Y Y$ & $r, 90$ & ש & $v, \ldots$ \\
\hline & 13 & $90-120$ & $\leq q, 7 r$ & $\leqslant r, \varepsilon$. & $v, q \vee$ & Loam & $\Lambda, \Upsilon^{\prime}$ & •, or & $1 \pi, \ldots$ & 11,70 & $1, v r$ & $\cdot, \operatorname{lr}$ & 0,90 \\
\hline & & $120-140$ & $\varepsilon \wedge, r_{0}$ & $\{0,1 \mu$ & $7,7 r$ & Loam & $\Lambda, r_{1}$ & $\cdot, \leqslant \vee$ & $1 \cdot, \Lambda$. & $1 ., 9$. & $1, v \cdot 7$ & $\cdot, 1 \leq$ & 0,11 \\
\hline & & W.P.M & $\leq \leqslant, \leqslant 7$ & rq,Or & $19, \cdot r$ & Loam & $\Lambda, \cdot 9$ & $\cdot, \vee \vee$ & 11,99 & $1 \cdot, \leqslant 0$ & $r, V V$ & $\cdot, 10$ & $9, .9$ \\
\hline & & $0-30$ & $r, r \cdot$ & $\varepsilon \cdot, \varepsilon r$ & $r \wedge, r \wedge$ & Clay Loam & 1,11 &., 01 & $r v, \varepsilon$. & $1 ., 10$ & $r, q V$ & $\cdot, Y_{1}$ & $19, \ldots$ \\
\hline & & $30-60$ & r, $0 \leqslant$ & $\{r, 1 \wedge$ & $r r, Y \wedge$ & Loam & $v, v$. & 1,11 & $r_{0}, \ldots$ & $1 \cdot, 11$ & $Y, T \leqslant$ & $\cdot, I V$ & $14,1$. \\
\hline & & $60-90$ & $r q, r q$ & $\leqslant v, 0$. & $1 \pi, 1 \leq$ & Loam & $\vee, \Lambda$. & $\cdot, 90$ & $1 ., 7$ & Ir,Tr & $r, r r r$ & $\cdot, 17$ & 9,1 . \\
\hline & 14 & $90-110$ & $\varepsilon r, v$. & or, r. & $r, v$ & Silt Loam & $V, \wedge r$ & $\cdot, V Y$ & $11, \ldots$ & $11,7$. & $1,0 \cdot 1$ & •, rr & $\wedge, 1$. \\
\hline & & $110-130$ & $\varepsilon V, r$. & $\varepsilon \cdot, \leqslant r$ & $\mid r, r \wedge$ & Loam & $\Lambda, r^{\prime}$ & $\cdot, r V$ & $9, \wedge$. & Ir,v. & 1,70 & - , ro & $7,0$. \\
\hline & & $130-150$ & $\varepsilon r, \varepsilon \cdot$ & $\leq 9,1 \leqslant$ & $V, \leq 7$ & Silt Loam & $\Lambda, r V$ & $\cdot, r q$ & 9.00 & $14,1$. & 1,70 & $\cdot, 17$ & $0, \cdot r$ \\
\hline & & W.P.M & $r \wedge, v r$ & $\{0,1 V$ & 17,1 . & Loam & $v, q v$ & $\cdot, v \cdot$ & 16.57 & 11,74 & $r, \leqslant r$ & $\cdot, 19$ & 11,09 \\
\hline & & $0-30$ & $\varepsilon Y, Y$ & $r r, O$. & $r \leqslant, q$ & Loam & $v, 71$ & $\cdot, Y \wedge$ & $r r, Y$. & 11,10 & $1, V \wedge r$ & $\cdot, 1 \leq$ & $1 \varepsilon, .1$ \\
\hline & & $30-60$ & $\varepsilon \varepsilon, r \wedge$ & $r v, 1 \leqslant$ & $1 \wedge, \leqslant \Lambda$ & Loam & $\vee, \wedge \vee$ & $\cdot, r V$ & $r \cdot \Lambda$. & Ir,O. & $1, v \cdot$ & $\cdot, 19$ & r \\
\hline & & $60-90$ & $\leqslant v, \ldots$ & \& & $1 \cdot, \wedge \mathrm{V}$ & Loam & $\vee, \wedge q$ & $\cdot, r q$ & IV, 7 . & Ir, & 1,7 & ( & $\Lambda, r \cdot$ \\
\hline & 15 & $90-120$ & $\varepsilon \Lambda, \vee \vee$ & $\varepsilon 0, \wedge$. & $0, \leqslant r$ & Sandy Loam & $\vee, \wedge q$ & $\cdot, r q$ & $1 r, \ldots$ & $11,0$. & $1, r r$ & $\cdot, Y_{0}$ & 0,11 \\
\hline & & $120-140$ & $\leqslant 0, \leqslant \wedge$ & $\leqslant 4, \leqslant$. & $\Lambda, 1 r$ & Loam & $\wedge, 10$ & $\cdot, \varepsilon$ & 8.40 & 11, r & $1,0.1$ & $\cdot, 11$ & $0, \ldots$ \\
\hline & & W.P.M & $\leqslant 0,74$ & $\varepsilon \cdot, r q$ & 14,90 & Loam & $\vee, \wedge T$ & • & 16.97 & 11,14 & 1,09 & $\cdot, 19$ & $9, \leqslant 9$ \\
\hline & & $0-30$ & $\leqslant 1, v_{0}$ & $r 1,10$ & $r v, 1$ & Clay Loam & $v, 71$ & $\cdot, Y Y$ & $r \wedge, r$. & $9, \wedge$. & $1, r Y$ & $\cdot, 1 \leq$ & $r \cdot, 0$. \\
\hline & & $30-60$ & $\{r, r \mid$ & $r_{0, r_{0}}$ & $r_{1}, 1 \leqslant$ & Clay Loam & $\vee, \vee \wedge$ & $\cdot, r r$ & $r v, \varepsilon$. & or & Y,YO & 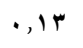 & $i v, \ldots$ \\
\hline & & $60-90$ & $\varepsilon \varepsilon, v \cdot$ & $r \cdot, v$. & $Y \leqslant, Y$. & Loam & $v, q$. & $\cdot, Y_{1}$ & $r_{\cdot}, q$. & 11,11 & $r, v \cdot r$ & $\cdot, 10$ & $v, \ldots$ \\
\hline & 16 & $90-120$ & $\left\{0, r_{0}\right.$ & $r_{0, r_{0}}$ & $19,8$. & Loam & $V, \wedge r$ & $\cdot, Y \leqslant$ & $r \cdot, V$. & Ir,o. & $r, r$. & $\cdot, 17$ & $\checkmark, \wedge$. \\
\hline & & $120-150$ & $\leqslant V, r \cdot$ & $r r, 1 \leqslant$ & $r \cdot, 74$ & Loam & $V, T V$ & $\cdot, Y_{0}$ & 15.30 & Ir, ro & 1,217 & $\cdot, I V$ & $\varepsilon, \wedge$ \\
\hline & & W.P.M & $\leqslant \leqslant, 0$. & $r \wedge, q Y$ & $r q, 0 \Lambda$ & Clay Loam & $V, V T$ & 0.24 & 22.50 & $11, r$. & $r, r Y$ & $\cdot, 10$ & $11, r r$ \\
\hline & & $0-30$ & $\leqslant 0, q$. & $r Y, \varepsilon r$ & $r 1,7 \Lambda$ & Sandy clay L & $V, 9 v$ & $\cdot, r r$ & $r_{\cdot}, r_{.}$ & $\mid r, 1$. & $Y, \otimes V$ & $\cdot, Y \leq$ & $1 \wedge, v$. \\
\hline & & $30-60$ & $\Delta r, \Delta V$ & $r 0,1 T$ & $r, r$. & Sandy clay $L$ & $v, q r$ & • & $1 \wedge, 0$. & Ir,oq & $r, q \cdot \varepsilon$ & $\cdot, 19$ & ४,०. \\
\hline & & $60-90$ & or,,$\wedge$ & $r V, 1 \leqslant$ & $19, .1$ & Sandy Loam & $v, q 1$ & $\cdot, Y^{4}$ & 17,7 & 14,11 & (r, & $\cdot, 1 r$ & $8, \ldots$ \\
\hline & 17 & $90-120$ & $0 \leqslant, Y T$ & r., & $10, \leqslant Y$ & Sandy Loam & $\vee, \wedge \wedge$ & • & $10, \varepsilon$. & $14,0$. & יזוזיז & $\cdot$, ir & $0,0$. \\
\hline & & $120-150$ & $0 \leqslant, 90$ & $r_{0}, \ldots$ & $1, . .0$ & Sandy Loam & $v, v_{0}$ & $\cdot, r$ & 12.90 & $i r, \Lambda$. & $\varepsilon, \pi$. & $\cdot, 10$ & $\varepsilon, r$. \\
\hline & & W.P.M & or,r. & $r \Lambda, \cdot 1$ & 19,79 & Loam & $\vee, \wedge q$ & $\cdot, r$ & 16.72 & $1 r, \cdot r$ & $r, r v$ & $\cdot, I V$ & $\wedge, 1 \wedge$ \\
\hline
\end{tabular}


M.S. Amira, et al.,

Table (3): Cont.

\begin{tabular}{|c|c|c|c|c|c|c|c|c|c|c|c|c|c|}
\hline \multirow{2}{*}{ 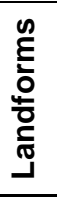 } & \multirow{2}{*}{$\begin{array}{l}\stackrel{o}{z} \\
\frac{0}{c} \\
\frac{0}{0} \\
\frac{0}{\alpha}\end{array}$} & \multirow{2}{*}{$\begin{array}{l}\text { Depth } \\
\text { Cm }\end{array}$} & \multicolumn{3}{|c|}{$\begin{array}{c}\text { Particle size } \\
\text { distribution \% }\end{array}$} & \multirow{2}{*}{ 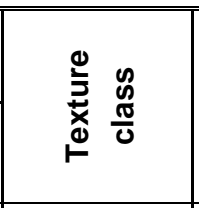 } & \multirow{2}{*}{ 돌 } & \multirow{2}{*}{ Ш } & \multirow{2}{*}{ 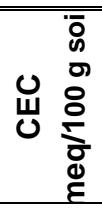 } & \multirow{2}{*}{ ESP } & \multirow{2}{*}{$\bigcup_{0}^{\infty} d^{\circ}$} & \multirow{2}{*}{$\sum_{0}^{\frac{\varepsilon}{2}} \overbrace{}^{\circ}$} & \multirow{2}{*}{$\begin{array}{l}\text { OM } \\
\text { g/kg }\end{array}$} \\
\hline & & & 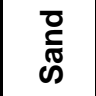 & $\frac{ \pm}{\bar{n}}$ & $\frac{\vec{\pi}}{U}$ & & & & & & & & \\
\hline \multirow{6}{*}{ 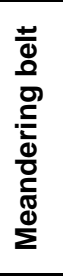 } & \multirow{6}{*}{18} & & or, O. & & $10, \ldots$ & Loam & $\vee, \vee \wedge$ & •, rr & $r \cdot, r$ & $r, \mid r$ & $1,1 \wedge \wedge$ & $\cdot, r)$ & $\cdot, 9$ \\
\hline & & $30-60$ & 71,90 & rr, & $\varepsilon, \wedge \vee$ & Sandy loam & $\vee, \vee \wedge$ & דוץ, & $\Lambda \Lambda, Y$. & $\mid r, 0$. & $\cdot, 99$ & . & $v, 00$ \\
\hline & & $60-90$ & $V V, T \leq$ & $1 \cdot, 1$. & $\mid r, Y 4$ & Sandy loam & $\vee, 9 \wedge$ & $\cdot, 19$ & $1 \leqslant, \wedge$. & $\mid r, q r$ & $\cdot, \Lambda_{0}$ & $\cdot, 19$ & $\varepsilon, 19$ \\
\hline & & $90-1$ & $\wedge \vee, r \vee$ & $0,0$. & $v, i r$ & Loamy Sand & $\wedge, 1 \leq$ & $\cdot, 11$ & $1 \cdot, r$. & $1 r, 1$. & דוד, & $\cdot, 17$ & $\varepsilon, 19$ \\
\hline & & 120 & 92.60 & 2.13 & 5.27 & Sand & 8.25 & 0.17 & 9.40 & 13.50 & 0.72 & 0.12 & 3.35 \\
\hline & & M & 74.41 & 16.68 & 8.91 & Sandy Loam & 7.99 & 0.21 & 14.58 & 12.83 & 0.88 & 0.16 & 6.038 \\
\hline \multirow{6}{*}{ 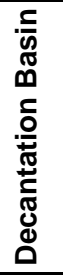 } & \multirow{6}{*}{19} & $0-30$ & $r r, q 0$ & $r_{0}, 1$. & $\varepsilon \cdot, 90$ & Clay & $\vee, \Lambda$. & $\cdot, r v$ & $r_{0,0}$. & $11,0$. & r, r & $\cdot, 11$ & $1 \wedge, 0$. \\
\hline & & $30-60$ & r & $r \cdot r$. & $r r, i v$ & Clay loam & $v, q 9$ & $\cdot, r \cdot$ & r., & $r, 1$. & 1,70 & $\cdot, I V$ & $9, \ldots$ \\
\hline & & $60-90$ & $r q, r$. & $r \Lambda, 1 r$ & $r r, O V$ & Loam & $\vee, q \vee$ & $\cdot, r V$ & rA,q. & $\mid r, r_{0}$ & $1, v_{1}$ & $\cdot, 11$ & $\wedge, 1$. \\
\hline & & $90-120$ & $r q, \ldots$ & $\leqslant r, 0$. & $11,0$. & Loam & $\vee, q \vee$ & $\cdot, r V$ & $r r, \ldots$ & Ir,Or & $r, T \varepsilon$ & $\cdot, I V$ & $V, r$. \\
\hline & & $120-150$ & $r \wedge, 0$. & 45.18 & 16.32 & Loam & 8.01 & 0.29 & 20.40 & 12.95 & 0.92 & 0.13 & 4.10 \\
\hline & & $\mathrm{M}$ & $r v, \leq \tau$ & 36.24 & 26.30 & Loam & 7.95 & 0.31 & 27.68 & 12.26 & 2.11 & 0.14 & 9.38 \\
\hline \multirow{6}{*}{$\begin{array}{l}\frac{0}{0} \\
\frac{d}{0} \\
\frac{0}{0} \\
0\end{array}$} & \multirow{6}{*}{20} & & $\leqslant 0, v$. & rr,o. & $r \cdot, \Lambda$ & Sand & $v, q 0$ & •, Yq & $r \leqslant, Y$. & $\mid r, 11$ & $r, 04$ & $\cdot, 10$ & $1 v .00$ \\
\hline & & & $\leqslant \vee, q$. & $r 0,11$ & r४, १৭ & Sandy clay L & $\wedge, 11$ & $\cdot, r q$ & $r_{\cdot}, r_{.}$ & $1 T, Y_{0}$ & $r, q \vee$ & $\cdot, 19$ & $\wedge, \ldots$ \\
\hline & & $60-5$ & $\varepsilon v, \varepsilon$. & $r \cdot, \ldots$ & $r Y, Y$ & & $\Lambda, I r$ & $\cdot, \leqslant 0$ & $19,1$. & $1 r, .0$ & 1,01 & $\cdot, 17$ & $8,9$. \\
\hline & & $90-120$ & $\varepsilon q, v_{0}$ & $r 1,1 r$ & $19,1 r$ & Loam & $\Lambda, \cdot r$ & $\cdot, 0 \leq$ & $11,0$. & $1 r, 0$. & 1,01 & $\cdot, 11$ & $0, \ldots$ \\
\hline & & $120-150$ & $\varepsilon \neg, Y_{0}$ & 35.00 & 18.75 & Loam & 8.23 & $\cdot, 0 \leqslant$ & 17.10 & $1 r, \Lambda_{0}$ & 1,70 & e,rr & $r, 0$. \\
\hline & & W.P.M & $\leqslant V, \varepsilon$. & 28.95 & 23.65 & Loam & 8.09 & $\cdot, \leqslant 0$ & 19.82 & Ir, ro & r, Yo & $\cdot, 11$ & $\Lambda, \cdot \Lambda$ \\
\hline \multirow{17}{*}{ ஓ } & \multirow{6}{*}{21} & $0-30$ & $r \leqslant, V_{0}$ & rv, ll & $\leqslant \Lambda, 1 \leqslant$ & Clay & $V, T V$ & ת r & $r_{0, r}$. & $10,0$. & 1,70 & • וr & Y४,A \\
\hline & & $30-70$ & $7, \vee \vee q$ & $\mu_{0,0}$. & $r, r_{1}$ & Sandy loam & $v, v$. & $\cdot, \pi$ & $r \bullet, r$. & $1 \leqslant, r$. & 1,70 & $\cdot, 11$ & $9,0$. \\
\hline & & $70-85$ & $\varepsilon \varepsilon, .9$ & $r v, 11$ & $1 \wedge, \wedge$ & Loani & $\vee, q \vee$ & $\cdot, \leqslant r$ & $r Y, V$. & $\mid r, Y \wedge$ & $\cdot, 99$ & $\cdot, I V$ & $\wedge, 7$. \\
\hline & & $85-120$ & $0 ., 0 \leqslant$ & $\leq 0, \ldots$ & $\{, \leqslant 4$ & Sandy loam & $v, 90$ & $\cdot, 7$ & $r \cdot, r$. & IY,Y. & $\cdot, 99$ & $\cdot, 17$ & $\vee, Y$. \\
\hline & & $120-150$ & $\varepsilon r, \diamond v$ & 40.40 & 16.03 & & 8.06 & 0.92 & 19.10 & 11.50 & 0.98 & 0.15 & 6.40 \\
\hline & & & $\varepsilon r, \wedge \mathrm{V}$ & 36.62 & 19.51 & Loam & 7.85 & 0.58 & 25.30 & 13.37 & 1.30 & 0.14 & 12.97 \\
\hline & \multirow{5}{*}{22} & & $\bullet \wedge, \bullet$. & $1 \cdot, 1$. & $r_{1}, \varepsilon$ & Sandy c & $v, v \vee$ & $\cdot, 19$ & $r q, v$. & IY,YO & $\cdot, 99$ & $\cdot, 11$ & $11, \varepsilon$ \\
\hline & & $30-60$ & $\Delta q, r \Delta$ & ir,o. & $r \wedge, 10$ & Sandy clay L & $v, q 0$ & $\cdot, 10$ & $r V, V$. & $M, \varepsilon \leq$ & $1, .0$ & •, Yr & $0, \cdot r$ \\
\hline & & $60-90$ & (4,q) & $10, \leqslant \leq$ & $r \cdot, r_{0}$ & Sandy clay L & 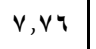 & $\cdot, 11$ & $r, 1$. & $1 r, 11$ & $1, .0$ & $\cdot, r \leq$ & $0, \cdot r$ \\
\hline & & $90-1$ & $77,9 \leq$ & $11, \leqslant \leq$ & $r 1, r r$ & Sandy c & $\vee, \Lambda$. & $\cdot, r_{0}$ & $r_{\cdot}, \cdot$ & $1 r, 0$. & 1.92 & 0.11 & 3.55 \\
\hline & & W.P.M & $M, O \leq$ & Ir, r. & $r 0,17$ & Sandy Clay L & $V, \wedge r$ & $\cdot, r \cdot$ & $r \leqslant, 0$. & $I Y, \wedge \Lambda$ & 1.30 & 0.18 & 7.66 \\
\hline & & $0-30$ & $\checkmark \wedge, r$. & $V, r r$ & $r \leqslant, r V$ & Sandy clay L & $v, v$. & ( & $r_{0}, \Lambda$. & $1 \cdot 1 \cdot 1 \cdot$ & 1,11 & $\cdot, 19$ & $1 \varepsilon, V$ \\
\hline & & $30-70$ & $79,7$. & $9,1$. & $r, r$. & Sandy clay L & $\vee, \wedge \vee$ & • & $r r, r$. & $1 ., 0$. & • & $\cdot, r_{0}$ & $\wedge, 0$. \\
\hline & & $70-90$ & $v_{0}, . \Lambda$ & $1,0$. & $1 \leqslant, \varepsilon r$ & Sandy loam & $\vee, q 9$ & $\cdot, r$. & $19,9$. & $|r| \leqslant$, &., 09 & $\cdot, 1 \leq$ & $r, 0$. \\
\hline & 23 & $90-110$ & $v \vee, \ldots$ & $1,0$. & $\mid r, 0$. & Sandy loam & $v, q \leq$ & $\cdot, 1 \leq$ & $\mid r, \ldots$ & $\mid r, 1$. & $\cdot, V r$ & r & $0, \cdot r$ \\
\hline & & $110-150$ & $\vee q, q$. & $9,0$. & $1 \cdot, 7$ & Sandy loam & $\vee, q r$ & $\cdot, r \wedge$ & $1 ., 9$. & Ir,o. & $1, \vee \wedge$ & $\cdot, 11$ & $0, \cdot r$ \\
\hline & & & $v r, \Lambda$. & $9, r r$ & $17,9 \vee$ & Sandy Loam & $\vee, \wedge \vee$ & $\cdot$, ro $_{0}$ & $I N, Y V$ & $11,0 r$ & $1, \cdot 7$ & $\cdot, 1 \mathrm{~V}$ & $v, 00$ \\
\hline & & & $r r, O V$ & $r \leqslant, r)$ & $\varepsilon r, r r$ & Clav & $V, T V$ & $\cdot, \lambda r$ & $r \wedge, v$ & Ir,o. & 1,70 & $\cdot, 10$ & $10,$. \\
\hline & & $30-60$ & $r \leqslant, Y$. & $r v, r$. & $r v, \Lambda$. & Clay loam & $\vee, \wedge 1$ & $\cdot, \varepsilon r$ & $r v, \Lambda$. & $\mid r, \wedge 0$ & $r, T \leq$ & $\cdot, I V$ & $Q, Y$. \\
\hline$\frac{\bar{Z}}{\sigma}$ & 24 & $60-90$ & $r q, \leqslant 0$ & $r \wedge, 0$. & $r r, .0$ & Clay loam & $\Lambda, \cdot V$ & $\cdot, 19$ & rr, & $\mid r, Y Y$ & $\cdot, 9 r$ & $\cdot, 17$ & $8,0$. \\
\hline$\underline{\underline{n}}$ & & $90-120$ & סر, וץ & $r q, 10$ & $r q, 0$. & Clay loam & $\Lambda, r$. & $\cdot, r q$ & $\curlyvee \wedge, \Lambda$. & ס & ५, १५ & $\cdot, 1 \leq$ & $\checkmark, \ldots$ \\
\hline & & $120-150$ & & $r q, 0$. & rV, 17 & Clay loam & $\Lambda, r Y$ & • & 17.60 & Ir,A. & 1,70 & • & $0, r$. \\
\hline & & W.P.M & $r \wedge, \leq \checkmark$ & $r v, v q$ & $r r, v_{0}$ & Clay Loam & $\Lambda,+1$ & - $\{1$ & 29.26 & $I r, r$. & $r, 17$ & $\cdot, 10$ & $\Lambda, \varepsilon$. \\
\hline
\end{tabular}


A recent geomorphic and pedological studies on Menouf province soils,

Table (4): Land capability indices and classes for the study area.

\begin{tabular}{|c|c|c|c|}
\hline \multirow{2}{*}{ Landform } & \multirow{2}{*}{ Soils of profile } & \multicolumn{2}{|c|}{ Land Capability } \\
\hline & & indices & classes \\
\hline \multirow{4}{*}{ High Terraces } & 1 & 56.66 & C3 (fair) \\
\hline & 2 & 51.93 & C3 (fair) \\
\hline & 3 & 52.46 & C3 (fair) \\
\hline & 4 & 56.30 & C3 (fair) \\
\hline \multirow{3}{*}{ Medium Terraces } & 5 & 67.00 & C2 (Good) \\
\hline & 6 & 65.80 & C2 (Good) \\
\hline & 7 & 53.52 & C3 (fair) \\
\hline \multirow{5}{*}{$\begin{array}{l}\text { Low } \\
\text { Terraces }\end{array}$} & 8 & 55.75 & C3 (fair) \\
\hline & 9 & 62.82 & C2 (Good) \\
\hline & 10 & 51.96 & C3 (fair) \\
\hline & 11 & 60.92 & C2 (Good) \\
\hline & 12 & 65.24 & C2 (Good) \\
\hline \multirow{5}{*}{ Over Flow Basin } & 13 & 60.26 & C2 (Good) \\
\hline & 14 & 60.10 & C2 (Good) \\
\hline & 15 & 55.22 & C3 (fair) \\
\hline & 16 & 66.31 & C2 (Good) \\
\hline & 17 & 62.69 & C2 (Good) \\
\hline Meandering Belt & 18 & 52.03 & C3 (fair) \\
\hline Decantation Basin & 19 & 66.94 & C2 (Good) \\
\hline Depression & 20 & 54.18 & C3 (fair) \\
\hline \multirow{3}{*}{ Levee } & 21 & 62.65 & C2 (Good) \\
\hline & 22 & 62.60 & C2 (Good) \\
\hline & 23 & $\mathbf{5 7 . 7 1}$ & C3 (fair) \\
\hline Island & 24 & 54.86 & C3 (fair) \\
\hline
\end{tabular}

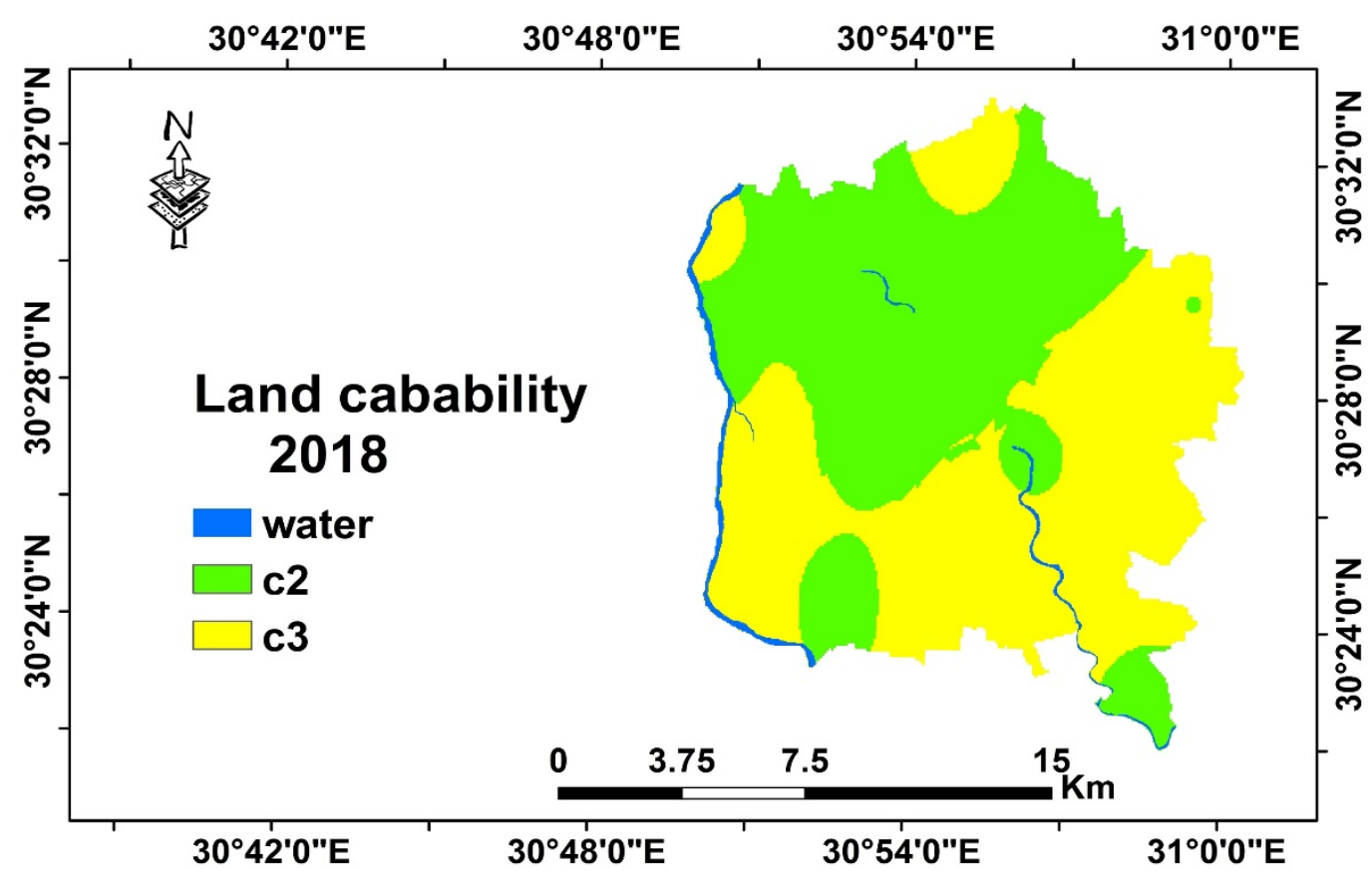

Fig (3): Land capability classes map of the studied area. 
M.S. Amira, et al.,

Table (5): Areas of land capability classes for the studied soils.

\begin{tabular}{|c|c|c|}
\hline \multirow{2}{*}{ Capability class } & \multicolumn{2}{|c|}{ Area } \\
\cline { 2 - 3 } & $\mathbf{k m}^{2}$ & $\%$ \\
\hline C2 (Good) & 107.16 & 47.63 \\
\hline C3 (Fair) & 117.84 & 52.37 \\
\hline
\end{tabular}

\section{REFERANCES}

Burt, Rebecca and Soil Survey Staff (2014). Kellogg Soil Survey Laboratory Methods Manual, Soil Survey Investigations Report No. 42, Version 5.0, Kellogg Soil Survey Laboratory, National Soil Survey Center, Natural Resources Conservation Service, USDA, Lincoln, Nebraska, USA.

CAPMS (2018). Egypt in figures, Central Agency for Pubic Mobilization and Statistics, https://www.capmas.gov.eg/Pages/Sta ticPages.aspx?page_id $=5035$.

Dehaan, R, L. and G. R. Taylor (2003). Image-derived spectral endmembers as indicators of salinization. International Journal of Remote Sensing, 24(4): 775-794.

Dobos, E., B. Norman, W. Bruee, M. Luca, J. Chris and M. Erika (2002). The use of DEM and satellite images for regional scale soil database. Proceedings of the 17th World Congress of Soil Science, Bangkok.

ESRI "Environmental Systems Research Institute" (2003): Using ArcGIS Geostatistical Analyst. Environmental Systems Research Institute (ESRI) Press, Redlands, California.
ESRI "Environmental Systems Research Institute" (2014): Arc Map Version 10.1 User Manual. ESRI, 380 New York Street, Redlands, California, 923738100, USA.

FAO (2006). Guidelines for soil profile description. Soil Res. Dev. and Co. Serv., Land and Water Dev. Div., Rome, Italy.

Ismail, H.A., M.H. Bahnassy and O.R. Abd El-Kawy (2005). Integrating GIS and modelling for agricultural land suitability evaluation at East Wadi ElNatrun, Egypt. Egyptian J Soil Sci., 45: 297-322.

ITT "International Telephone \& Telegraph" (2012). ITT corporation ENVI 5 software, 1133 Westchester Avenue, White Plains, NY 10604, USA.

Lillesand, T. M. and R. W. Kiefer (2007). Remote Sensing and Image Interpretation. 5th Ed. Paper back. John Wiley, New York.

Soil Survey Staff (2014). Keys to Soil Taxonomy, $11^{\text {th }}$ Ed., USDA, NRCS, Pocahontas Press, Inc., Blacksburg, Virginia, USA.

Zinck, J.A. and C.R. Valenzuela (1990): Soil Geographic Database: Structure and Application Examples. ITC journal, 3: 270. 


\section{دراسات جيومورفولوجية وبيدولوجية حديثة على أراضي مركز منوف، محافظة المنوفية، مصر}

محمد سمير عراقي عميرة(')، فوزي الثاذلي أبو عجوة(')، الحسيني عبد الغفار أبو حسين(')،

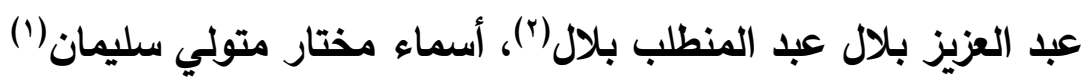

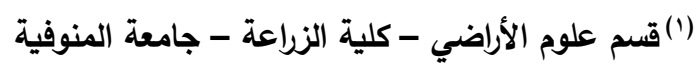

(†) الهيئة القومية للاستشعار من البعد وعلوم الفضاء - القاهرة

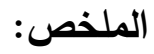

أجري هذا البحث خلال 1 1 ـ ب بهاف دراسة الخصائص الجيومرفولوجية والبيدولوجية وكذلك تقسيم وتقييم أراضي

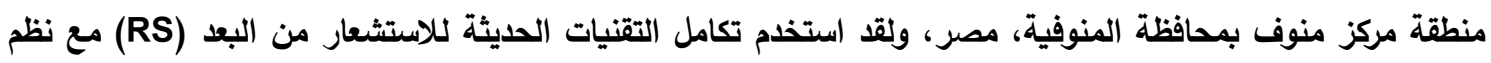
المعلومات الجغرافية (GIS) في اجراء هذا العمل. ولقد أوضحت الخريطة الجيومرفولوجية الناتجة من معالجة وتفسير الصورة الجوية أن منطقة الدارسة تتميز بوجود وحدة جيومرفولوجية رئيسية هي السهل الفيضي (Alluvial Plain) لرواسب دلتا نهر النيل التي يمكن تقسيمها إلى تسع أشكال

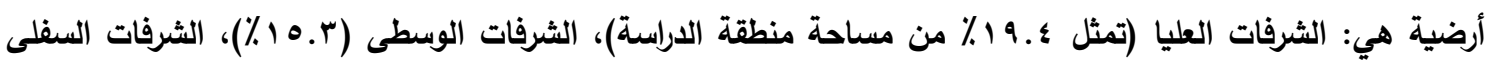

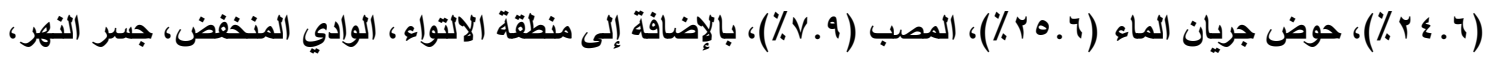
جزيرة (بمساحات محدودة). ولقد تم اختيار أربع وعشرون قطاعاً أرضياً لتمثل أراضي تلك الأثكال الأرضية، ودرست وسجلت الملامح الطبوغرافية

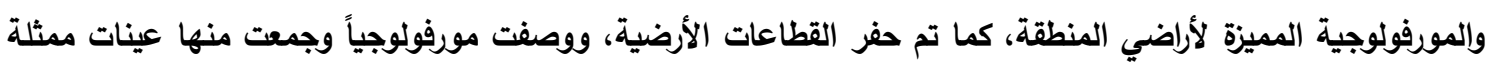
للأفاق المختلفة لإجراء التحليلات المعملية لتقدير الخواص الطبيعية والكيماوية.

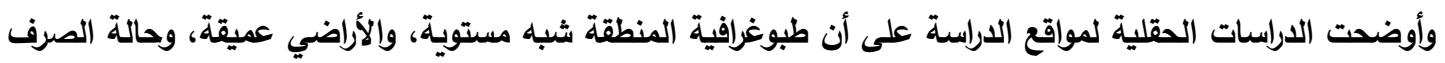

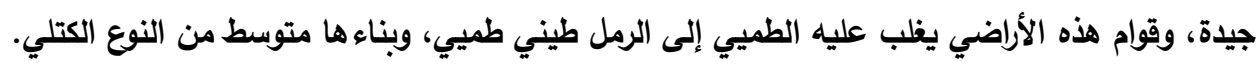

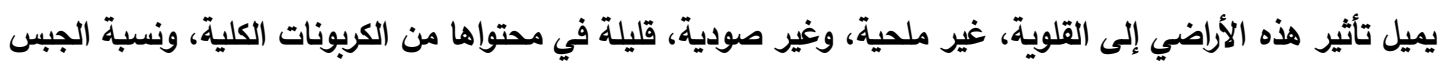

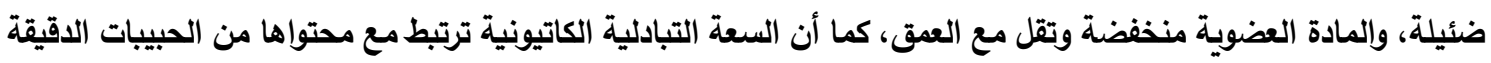

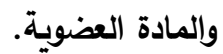
ونظراً لأنه لم يتضح في هذه الأراضي أي آفاق تشخيصية فلقد تم تقسيم تلك الأراضي تبعا للتقسيم الأمريكي الحديث

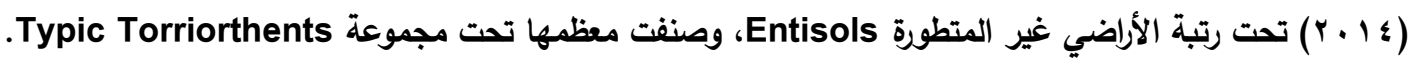

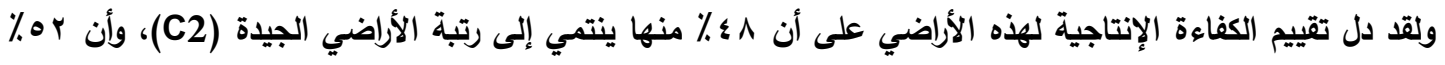
منها ينتمي إلى رتبة الأراضي المقبولة (C3). الكلمات الدالة: الاستشعار من البعد، نظم المعلومات الجغرافية، الوحدات الجيومورفولوجية، تقسيم الأراضي، تقييم الأراضي.

أ.د/ أحمد عبدالفتاح البارودى كلية الزراعة - جامعة طنطا

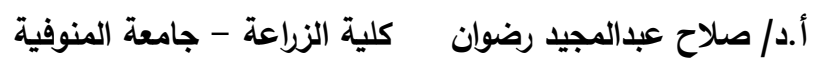


M.S. Amira, et al., 